\title{
Chemical Variability and In Vitro Anti-Inflammatory Activity of Leaf Essential Oil from Ivorian Isolona dewevrei (De Wild. \& T. Durand) Engl. \& Diels
}

\author{
Didjour Albert Kambiré ${ }^{1}$, Jean Brice Boti ${ }^{2}$, Ahmont Claude Landry Kablan ${ }^{1}$, Daouda Ballo ${ }^{2}$, Mathieu Paoli ${ }^{3}$ (D, \\ Virginie Brunini ${ }^{3}$ and Félix Tomi ${ }^{3, *(\mathbb{C})}$ \\ $1 \quad$ UPR de Chimie Organique, Département de Mathématiques, Physique et Chimie, UFR des Sciences \\ Biologiques, Université Péléforo Gon Coulibaly, Korhogo BP 1328, Côte d’Ivoire; \\ dakambire@gmail.com (D.A.K.); kablanahmont@yahoo.fr (A.C.L.K.) \\ 2 Laboratoire de Constitution et Réaction de la Matière, UFR-SSMT, Université Félix Houphouët-Boigny, \\ Abidjan BP 1328, Côte d'Ivoire; jeanbriceboti@hotmail.fr (J.B.B.); daoudaballo526@gmail.com (D.B.) \\ 3 Laboratoire Sciences Pour l'Environnement, Université de Corse-CNRS, UMR 6134 SPE, Route des \\ Sanguinaires, 20000 Ajaccio, France; paoli_m@univ-corse.fr (M.P.); brunini_v@univ-corse.fr (V.B.) \\ * Correspondence: tomi_f@univ-corse.fr
}

\section{check for}

updates

Citation: Kambiré, D.A. Boti, J.B.; Kablan, A.C.L.; Ballo, D.; Paoli, M.; Brunini, V.; Tomi, F. Chemical Variability and In Vitro Anti-Inflammatory Activity of Leaf Essential Oil from Ivorian Isolona dewevrei (De Wild. \& T. Durand) Engl. \& Diels. Molecules 2021, 26, 6228. https://doi.org/10.3390/molecules 26206228

Academic Editor: Isabelle Bombarda

Received: 15 September 2021

Accepted: 11 October 2021

Published: 15 October 2021

Publisher's Note: MDPI stays neutral with regard to jurisdictional claims in published maps and institutional affiliations.

Copyright: (c) 2021 by the authors. Licensee MDPI, Basel, Switzerland. This article is an open access article distributed under the terms and conditions of the Creative Commons Attribution (CC BY) license (https:// creativecommons.org/licenses/by/ $4.0 /)$
Abstract: The chemical variability and the in vitro anti-inflammatory activity of the leaf essential oil from Ivorian Isolona dewevrei were investigated for the first time. Forty-seven oil samples were analyzed using a combination of CC, GC(RI), GC-MS and ${ }^{13} \mathrm{C}-\mathrm{NMR}$, thus leading to the identification of 113 constituents (90.8-98.9\%). As the main components varied drastically from sample to sample, the 47 oil compositions were submitted to hierarchical cluster and principal components analyses. Three distinct groups, each divided into two subgroups, were evidenced. Subgroup I-A was dominated by $(Z)$ - $\beta$-ocimene, $\beta$-eudesmol, germacrene $\mathrm{D}$ and $(E)$ - $\beta$-ocimene, while $(10 \beta \mathrm{H})$ $1 \beta, 8 \beta$-oxido-cadina-4-ene, santalenone, trans- $\alpha$-bergamotene and trans- $\beta$-bergamotene were the main compounds of Subgroup I-B. The prevalent constituents of Subgroup II-A were germacrene $\mathrm{B},(E)$ - $\beta$-caryophyllene, $(5 \alpha \mathrm{H}, 10 \beta \mathrm{Me})-6,12$-oxido-elema-1,3,6,11(12)-tetraene and $\gamma$-elemene. Subgroup II-B displayed germacrene $\mathrm{B}$, germacrene $\mathrm{D}$ and $(Z)-\beta$-ocimene as the majority compounds. Germacrene D was the most abundant constituent of Group III, followed in Subgroup III-A by (E)- $\beta$-caryophyllene, $(10 \beta \mathrm{H})-1 \beta, 8 \beta$-oxido-cadina-4-ene, germacrene D-8-one, and then in Subgroup III-B by (Z)- $\beta$-ocimene and (E)- $\beta$-ocimene. The observed qualitative and quantitative chemical variability was probably due to combined factors, mostly phenology and season, then harvest site to a lesser extent. The lipoxygenase inhibition by a leaf oil sample was also evaluated. The oil $\mathrm{IC}_{50}$ $(0.020 \pm 0.005 \mathrm{mg} / \mathrm{mL})$ was slightly higher than the non-competitive lipoxygenase inhibitor NDGA $\mathrm{IC}_{50}(0.013 \pm 0.003 \mathrm{mg} / \mathrm{mL})$, suggesting a significant in vitro anti-inflammatory potential.

Keywords: Isolona dewevrei; leaf essential oil; chemical variability; in vitro anti-inflammatory activity

\section{Introduction}

Isolona Engl. is a genus belonging to the Annonaceae family and comprising about 20 species, widely distributed in tropical rain forests of West and Central Africa, and Madagascar. Five species of this genus grow wild in Côte d'Ivoire: I. cooperi, I. campanulata, I. soubreana, I. deightonii and I. dewevrei [1,2].

Isolona dewevrei (De Wild. \& T. Durand) Engl. \& Diels (synonym: Monodora dewevrei De Wild. \& T. Durand) is an evergreen shrub or a tree that can reach $15 \mathrm{~m}$ in height. It has narrowly obovate to obovate or elliptic to narrowly elliptic leaves that are $10-17 \mathrm{~cm}$ long and 4-7 cm wide, with an acuminated apex. The inflorescences appear on leafy branches and sometimes on older ones, whereas the fruits are ovoid $(6-7 \mathrm{~cm}$ long, $4-5 \mathrm{~cm}$ in diameter), smooth but very finely ribbed, glabrous, green and yellow at maturity [1]. Although no ethno-pharmacological use of $I$. dewevrei was reported in the literature, other 
Isolona species (I. cooperi and I. campanulata) are traditionally used as herbal medicine in Côte d'Ivoire to treat bronchial ailments, skin diseases, hematuria and infertility, and to facilitate childbirth [1,2].

Previous studies of some solvent extracts reported several alkaloids, sterols and sesquiterpenes, as well from I. maitlandii, I. cauliflora, I. pilosa, I. campanulata, I. zenkeri and I. hexaloba, as from I. cooperi, from Côte d'Ivoire [3-11]. The chemical composition of the leaf, stem bark, root bark essential oils from I. cooperi and leaf essential oil from I. campanulata were also determined. The leaf and stem bark oils from $I$. cooperi were dominated by (Z)- $\beta$-ocimene and $\gamma$-terpinene, while the prevalent compounds of the root bark were 5 -isopentenylindole and (E)- $\beta$-caryophyllene [12]. The leaf essential oil from $I$. campanulata was a sesquiterpene-rich oil. Its main compounds were either eudesm-5-en-11-ol, or (E)- $\beta$-caryophyllene and $\alpha$-humulene [13].

In our previous works, we investigated and reported for the first time the chemical composition of the leaf, root and stem bark essential oils from $I$. dewevrei. The structure of germacrene D-8-one, a new natural compound, was elucidated after isolation from the stem bark essential oil. The major compounds of the root oil were cyperene and camphene, followed by 5 -isopentenylindole, $\beta$-elemene and $(Z)$ - $\alpha$-bisabolene, whereas the stem bark oil was dominated by cyperene associated with $\beta$-elemene, $(Z)-\alpha-$ bisabolene, $(E)$ - $\beta$-caryophyllene and $\alpha$-copaene [14]. The leaf oil studies led to the isolation of seven new sesquiterpenes, characterized as 6,12-oxido-germacra-1(10),4,6,11(12)-tetraene ( $5 \alpha \mathrm{H}, 10 \beta \mathrm{Me})-6,12-$ oxido-elema-1,3,6,11(12)-tetraene, germacra-1(10),4,7(11)-trien-6,12- $\gamma$ lactone, $(1 \beta \mathrm{H}, 5 \beta \mathrm{H})-6,12-$ oxido-guaia-6,10(14),11(12)-trien-4 $\alpha$-ol, $(10 \beta \mathrm{H})-1 \beta, 8 \beta$-oxido-cadina4-ene, $(7 \beta \mathrm{H})$-germacrene $\mathrm{D}-8 \alpha$-ol and $(7 \beta \mathrm{H})$-germacrene $\mathrm{D}-8 \beta$-ol. NMR data of cadina$1(10), 4$-dien- $8 \beta$-ol were also reported for the first time $[15,16]$. The studied leaf oil samples evidenced three chemical compositions dominated either by germacrene $\mathrm{B},(5 \alpha \mathrm{H}, 10 \beta \mathrm{Me})$ 6,12-oxido-elema-1,3,6,11(12)-tetraene, germacrene $D,(Z)$ - $\beta$-ocimene, $\gamma$-elemene and (E)- $\beta$ caryophyllene, or by germacrene $\mathrm{D}$, germacrene $\mathrm{D}$-8-one, $(10 \beta \mathrm{H})-1 \beta, 8 \beta$-oxido-cadina-4ene, $(7 \beta H)$-germacrene D- $8 \alpha$-ol and cadina- $1(10), 4$-dien- $8 \beta$-ol, or by $(10 \beta \mathrm{H})-1 \beta, 8 \beta$-oxidocadina-4-ene, (E)- $\beta$-caryophyllene, germacrene D, cadina-1(10),4-dien-8 $\beta$-ol and caryophyllene oxide. Therefore, continuing our study on the chemical characterization of essential oils from Côte d'Ivoire [17-21], the chemical variability of the leaf essential oil of $I$. dewevrei was evaluated by investigating a larger number of oil samples (47). The in vitro antiinflammatory activity of this essential oil was tested too.

\section{Results and Discussion}

The chemical composition of 47 leaf essential oil samples from I. dewevrei growing wild in Côte d'Ivoire was investigated in order to highlight possible variability. Oil samples were isolated by the hydrodistillation of fresh leaves collected at six sites of the Bossématié forest (Eastern Côte d'Ivoire) and two sites of the Haut-Sassandra forest (Western Côte $\mathrm{d}$ 'Ivoire). The extraction yields calculated on a weight basis $(w / w)$ were in the range of $0.094-0.506 \%$. Analyses were carried out using a combination of GC(RI), GC-MS and ${ }^{13} \mathrm{C}-\mathrm{NMR}$, following a computerized method developed at the University of Corsica $[15,22]$. Most of the constituents were identified by the three techniques, including the identification by ${ }^{13} \mathrm{C}-\mathrm{NMR}$ of components present at a content as low as $0.4-0.5 \%$ and compiled in our laboratory-made ${ }^{13} \mathrm{C}$-NMR spectral data library. However, several minor compounds remained unidentified despite the use of these complementary techniques. Hence, samples S2 (4.115 g), S14 (4.820 g), S24 (3.702 g), S44 (4.226 g) and S47 (3.910 g), which displayed qualitative and quantitative variations of the main constituents and the minor unidentified compounds, were separately submitted to a detailed analysis by column chromatography (CC). The CC fractions were then analyzed by the three above techniques. Finally, the 47 oil compositions were subjected to a statistical analysis, and the in vitro anti-inflammatory activity of the single monoterpene-rich oil sample (S44) was evaluated. 


\subsection{Detailed Analysis of Essential Oil Samples S2, S14, S24, S44 and S47}

The leaf oil samples S2, S14, S24, S44 and S47 were representative of the whole sampling and displayed different chromatographic profiles with several unidentified minor components. They were separately fractionated by silica gel column chromatography, and seven fractions were eluted for each sample using a gradient of solvents, $n$-pentane/diethyl ether. Fractions F1 and F2 contained non-polar compounds, while medium polar components were in fractions F3-F6 and polar constituents in fraction F7. The CC fractions analysis by GC(RI), GC-MS and ${ }^{13} \mathrm{C}-\mathrm{NMR}$ led to the identification of various minor constituents along with the majority compounds of the samples

Special attention was paid to the identification of some compounds. For instance, eight sesquiterpenes previously isolated and characterized from the leaf essential oil of I. dewerrei were identified by their reported ${ }^{13} \mathrm{C}-\mathrm{NMR}$ data and retention indices [15,16]: $(5 \alpha \mathrm{H}, 10 \beta \mathrm{Me})-6,12$-oxido-elema-1,3,6,11(12)-tetraene (fractions F3 from samples S14 and S47; 48.5 and $39.2 \%$, respectively), 6,12-oxido-germacra-1(10),4,6,11(12)-tetraene (fraction F3

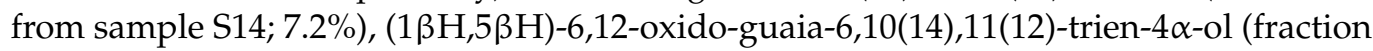
F5 from sample S14; 56.4\%), germacra-1(10),4,7(11)-trien-6,12- $\gamma$-lactone (fraction F6 from sample S14; 19.3\%), (10 $3 \mathrm{H})$-1 $\beta, 8 \beta$-oxido-cadina-4-ene (fraction F4 from samples S2, S24 and $\mathrm{S} 44 ; 34.7,18.6$ and $14.7 \%$, respectively), $(7 \beta \mathrm{H})$-germacrene D-8 $\alpha$-ol (fraction F5 from sample S24; $29.4 \%),(7 \beta \mathrm{H})$-germacrene D-8 $\beta$-ol (fraction F5 from sample S24; $9.8 \%$ ) and cadina-1(10),4-dien-8 $\beta$-ol (fraction F5 from sample S24;39.2\%). Similarly, germacrene D-8one previously isolated from the stem bark essential oil of the same species was identified in fraction F3 from sample S24 (28.6\%) [14].

Compounds bearing the elemane and germacrane skeletons and those co-eluting during the GC analysis needed special attention too. Indeed, under GC and GC-MS thermal conditions, germacrene compounds bearing the cyclodeca-1,5-diene sub-structure partially or totally rearranged to the corresponding elemanes (Cope transposition) [23,24]. This was the case for 6,12-oxido-germacra-1(10),4,6,11(12)-tetraene and (5 $\alpha \mathrm{H}, 10 \beta \mathrm{Me})-6,12$ oxido-elema-1,3,6,11(12)-tetraene, and then germacrene B and $\gamma$-elemene. Therefore, their correct contents were obtained by a combination of GC(FID) and ${ }^{13} \mathrm{C}-\mathrm{NMR}$ as previously reported $[15,16]$. However, $\delta$-elemene and $\beta$-elemene were really secondary metabolites produced by the plant and not rearranged products since germacrene $A$ and germacrene $C$ were not detected by ${ }^{13} \mathrm{C}-\mathrm{NMR}$ in the samples. The diastereoisomers $(7 \beta \mathrm{H})$-germacrene D- $8 \alpha$-ol and $(7 \beta \mathrm{H})$-germacrene D-8 $\beta$-ol co-eluted on apolar and polar columns during the GC analysis. Thus, the ${ }^{13} \mathrm{C}-\mathrm{NMR}$ analysis allowed their identification, and their relative contents were assessed by a combination of GC(FID) and ${ }^{13} \mathrm{C}-\mathrm{NMR}[15,16]$.

The detailed analysis of essential oil samples S2, S14, S24, S44 and S47, by a combination of chromatographic and spectroscopic techniques led to the identification of a total of 99 compounds representing, respectively, $97.8,96.4,93.8,98.9$ and $98.1 \%$ of their whole composition (Table 1). Oil samples S2, S14, S24 and S47 were dominated by sesquiterpenes (76.0-92.5\%), whereas sample S44 was monoterpene rich (71.2\%). Significant variations appeared relative to the majority compounds. Indeed, the main constituents of oil sample S2 were santalenone $(13.0 \%)$, trans- $\alpha$-bergamotene $(11.7 \%)$, trans- $\beta$-bergamotene $(10.9 \%)$, $(10 \beta \mathrm{H})-1 \beta, 8 \beta$-oxido-cadina- 4 -ene (9.3\%), $\beta$-bisabolene $(8.7 \%)$ and $\alpha$-santalene ( $8.5 \%)$. Sample S14 was dominated by germacrene B $(20.8 \%)$, followed by $(5 \alpha \mathrm{H}, 10 \beta \mathrm{Me})-6,12$-oxidoelema-1,3,6,11(12)-tetraene (10.3\%), (Z)- $\beta$-ocimene $(7.8 \%)$, germacrene D $(7.5 \%), \gamma$-elemene $(6.9 \%)$ and $(E)-\beta$-caryophyllene (6.8\%). Germacrene $\mathrm{D}(23.6 \%)$, germacrene $\mathrm{D}-8$-one $(8.7 \%)$, $(7 \beta \mathrm{H})$-germacrene D-8- $\alpha$-ol (7.8\%), cadina-1(10),4-dien- $8 \beta$-ol $(7.6 \%)$ and $(10 \beta \mathrm{H})-1 \beta, 8 \beta$ oxido-cadina-4-ene (7.3\%) were the most prevalent constituents of sample S24. Sample S44 was largely dominated by $(Z)-\beta$-ocimene $(36.6 \%)$ and $(E)$ - $\beta$-ocimene $(30.5 \%)$, followed by germacrene $\mathrm{D}(13.8 \%)$, while $\beta$-eudesmol $(22.5 \%)$, germacrene $\mathrm{D}(16.3 \%), \alpha$-eudesmol $(12.4 \%),(E)-\beta$-caryophyllene (12.4\%) and (Z)- $\beta$-ocimene (10.2\%) were the main compounds of sample S47. Obviously, the chemical compositions of the five essential oil samples displayed qualitative and quantitative variations (Table 1). 
Table 1. Chemical composition of five leaf essential oil samples from Isolona dewevrei.

\begin{tabular}{|c|c|c|c|c|c|c|c|c|c|c|c|}
\hline $\mathbf{N}^{\circ}$ & Compounds ${ }^{a}$ & $\mathrm{RII}^{\mathrm{b}}$ & RIa & RIp & RRF & S2 (\%) & S14 (\%) & S24 (\%) & S44 (\%) & S47 (\%) & Identification \\
\hline 1 & $\alpha$-Thujene & 932 & 923 & 1016 & 0.765 & 0.2 & 0.5 & $\operatorname{tr}$ & 0.1 & 0.2 & $\begin{array}{c}\text { RI, MS, } \\
{ }^{13} \text { C-NMR }\end{array}$ \\
\hline 2 & $\alpha$-Pinene & 936 & 931 & 1013 & 0.765 & 0.1 & 0.4 & 0.1 & $\operatorname{tr}$ & 0.1 & $\begin{array}{c}\text { RI, MS, } \\
{ }^{13} \text { C-NMR }\end{array}$ \\
\hline 3 & Sabinene & 973 & 965 & 1120 & 0.765 & 0.6 & 1.8 & 0.1 & 0.2 & 1.0 & $\begin{array}{c}\text { RI, MS, } \\
{ }^{13} \text { C-NMR }\end{array}$ \\
\hline 4 & $\beta$-Pinene & 978 & 970 & 1109 & 0.765 & $\operatorname{tr}$ & 0.4 & 0.1 & - & - & $\begin{array}{c}\text { RI, MS, } \\
{ }^{13} \text { C-NMR }\end{array}$ \\
\hline 5 & Myrcene & 987 & 981 & 1158 & 0.765 & 0.2 & 0.6 & 0.3 & 0.9 & 0.3 & $\begin{array}{c}\text { RI, MS, } \\
{ }^{13} \text { C-NMR }\end{array}$ \\
\hline 6 & $\alpha$-Phellandrene & 1002 & 997 & 1162 & 0.765 & $\operatorname{tr}$ & 0.1 & $\operatorname{tr}$ & 0.1 & - & RI, MS \\
\hline 7 & $\delta$-3-Carene & 1010 & 1005 & 1146 & 0.765 & $\operatorname{tr}$ & 0.1 & $\operatorname{tr}$ & 0.1 & - & RI, MS \\
\hline 8 & $\alpha$-Terpinene & 1013 & 1009 & 1178 & 0.765 & $\operatorname{tr}$ & 0.1 & 0.1 & 0.2 & $\operatorname{tr}$ & $\begin{array}{c}\text { RI, MS, } \\
{ }^{13} \text { C-NMR }\end{array}$ \\
\hline 9 & p-Cymene & 1015 & 1012 & 1268 & 0.698 & 0.4 & 0.3 & $\operatorname{tr}$ & 0.1 & - & $\begin{array}{c}\text { RI, MS, } \\
{ }^{13} \text { C-NMR }\end{array}$ \\
\hline 10 & Limonene & 1025 & 1021 & 1199 & 0.765 & 0.1 & 2.4 & 1.1 & 0.2 & $\operatorname{tr}$ & $\begin{array}{c}\text { RI, MS, } \\
{ }^{13} \text { C-NMR }\end{array}$ \\
\hline 11 & (Z)- $\beta$-Ocimene & 1029 & 1025 & 1230 & 0.765 & 0.8 & 7.8 & 3.4 & 36.6 & 10.2 & $\begin{array}{c}\text { RI, MS, } \\
{ }^{13} \text { C-NMR }\end{array}$ \\
\hline 12 & $(E)$ - $\beta$-Ocimene & 1041 & 1036 & 1247 & 0.765 & 0.5 & 4.8 & 4.5 & 30.5 & 3.4 & $\begin{array}{c}\text { RI, MS, } \\
{ }^{13} \text { C-NMR }\end{array}$ \\
\hline 13 & $\gamma$-Terpinene & 1051 & 1048 & 1242 & 0.765 & 0.2 & 0.3 & 0.2 & 0.5 & 0.1 & $\begin{array}{c}\text { RI, MS, } \\
{ }^{13} \text { C-NMR }\end{array}$ \\
\hline 14 & Terpinolene & 1082 & 1078 & 1279 & 0.765 & $\operatorname{tr}$ & 0.1 & $\operatorname{tr}$ & 0.1 & - & RI, MS \\
\hline 15 & Linalool & 1086 & 1083 & 1543 & 0.869 & 0.1 & 0.2 & $\operatorname{tr}$ & 0.1 & $\operatorname{tr}$ & $\begin{array}{c}\text { RI, MS, } \\
{ }^{13} C-N M R\end{array}$ \\
\hline 16 & allo-Ocimene & 1113 & 1117 & 1370 & 0.765 & 0.1 & 0.2 & 0.1 & 1.3 & 0.4 & $\begin{array}{c}\text { RI, MS, } \\
{ }^{13} \text { C-NMR }\end{array}$ \\
\hline 17 & Terpinen-4-ol & 1164 & 1161 & 1597 & 0.869 & 0.1 & 0.2 & - & $\operatorname{tr}$ & 0.1 & $\begin{array}{c}\text { RI, MS, } \\
{ }^{13} C-N M R\end{array}$ \\
\hline 18 & Neral & 1215 & 1212 & 1679 & 0.887 & - & 0.1 & - & $\operatorname{tr}$ & 0.1 & RI, MS \\
\hline 19 & Geraniol & 1235 & 1233 & 1843 & 0.869 & 0.2 & - & 0.1 & $\operatorname{tr}$ & $\operatorname{tr}$ & $\begin{array}{c}\text { RI, MS, } \\
{ }^{13} \text { C-NMR }\end{array}$ \\
\hline 20 & Geranial & 1244 & 1244 & 1740 & 0.887 & $\operatorname{tr}$ & $\operatorname{tr}$ & 0.1 & - & 0.1 & RI, MS \\
\hline 21 & Thymol & 1267 & 1267 & 2178 & 0.808 & 1.7 & - & $\operatorname{tr}$ & 0.2 & - & $\begin{array}{c}\text { RI, MS, } \\
{ }^{13} \text { C-NMR }\end{array}$ \\
\hline 22 & Carvacrol & 1278 & 1277 & 2219 & 0.808 & - & $\operatorname{tr}$ & - & - & 1.0 & $\begin{array}{c}\text { RI, MS, } \\
{ }^{13} \text { C-NMR }\end{array}$ \\
\hline 23 & Eugenol & 1331 & 1328 & 2170 & 0.808 & - & - & - & - & 0.4 & $\begin{array}{c}\text { RI, MS, } \\
{ }^{13} \text { C-NMR }\end{array}$ \\
\hline 24 & Bicycloelemene & 1338 & 1331 & 1485 & 0.751 & - & 0.2 & - & - & 0.1 & $\begin{array}{c}\text { RI, MS, } \\
{ }^{13} C-N M R\end{array}$ \\
\hline 25 & $\delta$-Elemene & 1340 & 1334 & 1464 & 0.751 & - & 3.9 & $\operatorname{tr}$ & 0.3 & 1.9 & $\begin{array}{c}\text { RI, MS, } \\
{ }^{13} \text { C-NMR }\end{array}$ \\
\hline 26 & $\alpha$-Cubebene & 1355 & 1347 & 1452 & 0.751 & - & 0.1 & 0.1 & 0.2 & - & $\begin{array}{c}\text { RI, MS, } \\
{ }^{13} \text { C-NMR }\end{array}$ \\
\hline 27 & $\alpha$-Ylangene & 1376 & 1368 & 1475 & 0.751 & - & 0.1 & $\operatorname{tr}$ & 0.1 & 0.1 & RI, MS \\
\hline 28 & $\alpha$-Copaene & 1379 & 1374 & 1485 & 0.751 & - & 0.2 & 0.9 & 0.8 & 0.1 & $\begin{array}{c}\text { RI, MS, } \\
{ }^{13} \text { C-NMR }\end{array}$ \\
\hline 29 & $\beta$-Cubebene & 1390 & 1384 & 1539 & 0.751 & 0.7 & - & - & 0.3 & 0.1 & $\begin{array}{c}\text { RI, MS, } \\
{ }^{13} \text { C-NMR }\end{array}$ \\
\hline 30 & $\beta$-Elemene & 1389 & 1385 & 1583 & 0.751 & $\operatorname{tr}$ & 3.1 & 1.6 & 1.3 & 1.2 & $\begin{array}{c}\text { RI, MS, } \\
{ }^{13} \text { C-NMR }\end{array}$ \\
\hline 31 & $\alpha$-Funebrene & 1385 & 1386 & 1518 & 0.751 & 0.6 & - & - & $\operatorname{tr}$ & - & $\begin{array}{c}\text { RI, MS, } \\
{ }^{13} \text { C-NMR }\end{array}$ \\
\hline 32 & Sesquithujene & 1399 & 1400 & 1549 & 0.751 & 0.2 & $\operatorname{tr}$ & - & $\operatorname{tr}$ & - & $\begin{array}{c}\text { RI, MS, } \\
{ }^{13} \text { C-NMR }\end{array}$ \\
\hline
\end{tabular}


Table 1. Cont.

\begin{tabular}{|c|c|c|c|c|c|c|c|c|c|c|c|}
\hline $\mathbf{N}^{\circ}$ & Compounds $^{a}$ & RII $^{b}$ & RIa & RIp & RRF & S2 (\%) & S14 (\%) & S24 (\%) & S44 (\%) & S47 (\%) & Identification \\
\hline 33 & Cyperene & 1402 & 1404 & 1524 & 0.751 & - & 0.3 & - & - & $\operatorname{tr}$ & $\begin{array}{c}\text { RI, MS, } \\
{ }^{13} \text { C-NMR }\end{array}$ \\
\hline 34 & cis- $\alpha$-Bergamotene & 1411 & 1409 & 1561 & 0.751 & 2.3 & $\operatorname{tr}$ & - & - & - & $\begin{array}{c}\text { RI, MS, } \\
{ }^{13} \text { C-NMR }\end{array}$ \\
\hline 35 & $\begin{array}{c}(E)-\beta- \\
\text { Caryophyllene }_{*}\end{array}$ & 1421 & 1416 & 1589 & 0.751 & 1.7 & 6.8 & 5.0 & 2.2 & 12.4 & $\begin{array}{c}\text { RI, MS, } \\
{ }^{13} \text { C-NMR }\end{array}$ \\
\hline 36 & $\alpha$-Santalene ${ }^{*}$ & 1422 & 1416 & 1565 & 0.751 & 8.5 & $\operatorname{tr}$ & 0.1 & - & - & $\begin{array}{c}\text { RI, MS, } \\
{ }^{13} \text { C-NMR }\end{array}$ \\
\hline 37 & $\beta$-Copaene * & 1430 & 1426 & 1574 & 0.751 & 0.3 & - & - & 0.2 & 0.1 & $\begin{array}{c}\text { RI, MS, } \\
{ }^{13} \text { C-NMR }\end{array}$ \\
\hline 38 & $\gamma$-Elemene ${ }^{*} \#$ & 1429 & 1426 & 1630 & 0.751 & - & 6.9 & $\operatorname{tr}$ & $\operatorname{tr}$ & 0.3 & $\begin{array}{c}\text { RI, MS, } \\
{ }^{13} \text { C-NMR }\end{array}$ \\
\hline 39 & $\begin{array}{c}\text { trans- } \alpha- \\
\text { Bergamotene }\end{array}$ & 1434 & 1431 & 1578 & 0.751 & 11.7 & - & - & $\operatorname{tr}$ & $\operatorname{tr}$ & $\begin{array}{c}\text { RI, MS, } \\
{ }^{13} \text { C-NMR }\end{array}$ \\
\hline 40 & Sesquisabinene A & 1435 & 1434 & 1636 & 0.751 & 1.3 & - & - & - & $\operatorname{tr}$ & $\begin{array}{c}\text { RI, MS, } \\
{ }^{13} \text { C-NMR }\end{array}$ \\
\hline 41 & $\beta$-Sesquifenchene & 1437 & 1439 & 1611 & 0.751 & 0.5 & - & $\operatorname{tr}$ & - & - & $\begin{array}{c}\text { RI, MS, } \\
{ }^{13} \text { C-NMR }\end{array}$ \\
\hline 42 & epi- $\beta$-Santalene & 1446 & 1441 & 1626 & 0.751 & 0.5 & 0.1 & $\operatorname{tr}$ & - & - & $\begin{array}{c}\text { RI, MS, } \\
{ }^{13} \text { C-NMR }\end{array}$ \\
\hline 43 & $(E)$ - $\beta$-Farnesene & 1446 & 1446 & 1660 & 0.751 & 2.1 & 0.1 & 0.1 & $\operatorname{tr}$ & - & $\begin{array}{c}\text { RI, MS, } \\
{ }^{13} \text { C-NMR }\end{array}$ \\
\hline 44 & $\alpha$-Humulene & 1455 & 1448 & 1662 & 0.751 & 0.8 & 1.2 & 1.3 & 0.5 & 1.9 & $\begin{array}{c}\text { RI, MS, } \\
{ }^{13} \text { C-NMR }\end{array}$ \\
\hline 45 & $\beta$-Santalene & 1460 & 1453 & 1643 & 0.751 & 0.2 & - & $\operatorname{tr}$ & - & - & $\begin{array}{c}\text { RI, MS, } \\
{ }^{13} C-N M R\end{array}$ \\
\hline 46 & $\begin{array}{c}(5 \alpha \mathrm{H}, 10 \beta \mathrm{Me})-6,12- \\
\text { Oxido- } \\
\text { elema-1,3,6,11(12)- } \\
\text { tetraene } \\
\#\end{array}$ & $1455^{\mathrm{c}}$ & 1455 & 1837 & 0.853 & - & 10.3 & 0.1 & - & 1.8 & $\begin{array}{c}\text { RI, MS, } \\
{ }^{13} \text { C-NMR }\end{array}$ \\
\hline 47 & Ishwarane & 1468 & 1460 & 1644 & 0.751 & 0.2 & - & - & - & - & $\begin{array}{c}\text { RI, MS, } \\
{ }^{13} C-N M R\end{array}$ \\
\hline 48 & $\beta$-Acoradiene & 1465 & 1461 & 1669 & 0.751 & 0.2 & - & - & $\operatorname{tr}$ & - & $\begin{array}{c}\text { RI, MS, } \\
{ }^{13} C-N M R\end{array}$ \\
\hline 49 & $\begin{array}{c}\text { 6,12-Oxido- } \\
\text { germacra- } \\
\text { 1(10),4,6,11(12)- } \\
\text { tetraene } \\
\#\end{array}$ & $1463^{c}$ & 1463 & 1845 & 0.853 & - & 1.0 & - & - & 0.2 & $\begin{array}{c}\text { RI, MS, } \\
{ }^{13} \text { C-NMR }\end{array}$ \\
\hline 50 & $\alpha$-Curcumene & 1473 & 1469 & 1766 & 0.707 & 1.1 & 0.2 & $\operatorname{tr}$ & $\operatorname{tr}$ & - & $\begin{array}{c}\text { RI, MS, } \\
{ }^{13} \text { C-NMR }\end{array}$ \\
\hline 51 & $\gamma$-Muurolene & 1474 & 1471 & 1683 & 0.751 & 0.8 & 2.5 & 0.3 & 0.3 & 0.2 & $\begin{array}{c}\text { RI, MS, } \\
{ }^{13} \text { C-NMR }\end{array}$ \\
\hline 52 & Germacrene D & 1479 & 1474 & 1700 & 0.751 & 0.2 & 7.5 & 23.6 & 13.8 & 16.3 & $\begin{array}{c}\text { RI, MS, } \\
{ }^{13} \text { C-NMR }\end{array}$ \\
\hline 53 & $\begin{array}{c}\text { trans- } \beta- \\
\text { Bergamotene }\end{array}$ & 1480 & 1478 & 1676 & 0.751 & 10.9 & 0.5 & $\operatorname{tr}$ & - & - & $\begin{array}{c}\text { RI, MS, } \\
{ }^{13} \text { C-NMR }\end{array}$ \\
\hline 54 & $\beta$-Selinene & 1486 & 1484 & 1710 & 0.751 & 0.1 & 0.1 & 0.1 & $\operatorname{tr}$ & 1.8 & $\begin{array}{c}\text { RI, MS, } \\
{ }^{13} \text { C-NMR }\end{array}$ \\
\hline 55 & $\alpha$-Zingiberene & 1489 & 1485 & 1712 & 0.751 & 0.4 & - & - & 0.1 & - & $\begin{array}{c}\text { RI, MS, } \\
{ }^{13} \text { C-NMR }\end{array}$ \\
\hline 56 & $\alpha$-Selinene & 1494 & 1490 & 1723 & 0.751 & 0.1 & - & - & - & 1.4 & $\begin{array}{c}\text { RI, MS, } \\
{ }^{13} \text { C-NMR }\end{array}$ \\
\hline 57 & Bicyclogermacrene * & 1494 & 1491 & 1721 & 0.751 & 0.1 & 0.1 & 1.8 & 0.7 & - & $\begin{array}{c}\text { RI, MS, } \\
{ }^{13} \text { C-NMR }\end{array}$ \\
\hline 58 & $\alpha$-Muurolene ${ }^{*}$ & 1496 & 1491 & 1720 & 0.751 & $\operatorname{tr}$ & - & - & 0.2 & 0.1 & $\begin{array}{c}\text { RI, MS, } \\
{ }^{13} C-N M R\end{array}$ \\
\hline 59 & $(Z)-\alpha$-Bisabolene & 1494 & 1492 & 1724 & 0.751 & 0.1 & 0.1 & - & - & - & RI, MS \\
\hline
\end{tabular}


Table 1. Cont.

\begin{tabular}{|c|c|c|c|c|c|c|c|c|c|c|c|}
\hline $\mathbf{N}^{\circ}$ & Compounds $^{a}$ & $\mathbf{R I I}^{\mathbf{b}}$ & RIa & RIp & RRF & S2 (\%) & S14 (\%) & S24 (\%) & S44 (\%) & S47 (\%) & Identification \\
\hline 60 & $\gamma$-Cadinene & 1507 & 1493 & 1753 & 0.751 & - & 0.1 & 0.2 & 0.1 & 0.1 & $\begin{array}{c}\text { RI, MS, } \\
{ }^{13} \mathrm{C}-\mathrm{NMR}\end{array}$ \\
\hline 61 & $\beta$-Bisabolene & 1503 & 1500 & 1719 & 0.751 & 8.7 & 0.1 & 0.2 & $\operatorname{tr}$ & - & $\begin{array}{c}\text { RI, MS, } \\
{ }^{13} \text { C-NMR }\end{array}$ \\
\hline 62 & $(E, E)$ - $\alpha$-Farnesene & 1498 & 1501 & 1748 & 0.751 & 0.7 & 0.3 & - & - & - & $\begin{array}{c}\text { RI, MS, } \\
{ }^{13} \text { C-NMR }\end{array}$ \\
\hline 63 & $\beta$-Curcumene & 1503 & 1502 & 1736 & 0.751 & 0.4 & - & - & - & - & $\begin{array}{c}\text { RI, MS, } \\
{ }^{13} \text { C-NMR }\end{array}$ \\
\hline 64 & (Z)- $\gamma$-Curcumene & 1493 & 1506 & 1732 & 0.751 & 0.8 & 0.2 & - & - & - & $\begin{array}{c}\text { RI, MS, } \\
{ }^{13} \text { C-NMR }\end{array}$ \\
\hline 65 & $\begin{array}{c}\beta- \\
\text { Sesquiphellandrene }\end{array}$ & 1516 & 1512 & 1766 & 0.751 & 0.4 & - & - & - & - & $\begin{array}{c}\text { RI, MS, } \\
{ }^{13} \text { C-NMR }\end{array}$ \\
\hline 66 & $\delta$-Cadinene & 1520 & 1514 & 1753 & 0.751 & - & $\operatorname{tr}$ & 2.5 & 0.8 & 0.3 & $\begin{array}{c}\text { RI, MS, } \\
{ }^{13} \text { C-NMR }\end{array}$ \\
\hline 67 & cis-Lanceol & $1525^{\mathrm{d}}$ & 1517 & 2087 & 0.819 & 0.3 & $\operatorname{tr}$ & 0.9 & 0.2 & - & $\begin{array}{c}\text { RI, MS, } \\
{ }^{13} \text { C-NMR }\end{array}$ \\
\hline 68 & (Z)- $\gamma$-Bisabolene & 1505 & 1521 & 1721 & 0.751 & 0.9 & 0.6 & 1.4 & 0.2 & - & $\begin{array}{c}\text { RI, MS, } \\
{ }^{13} \text { C-NMR }\end{array}$ \\
\hline 69 & $\begin{array}{c}\text { trans- } \\
\text { Sesquisabinene } \\
\text { hydrate }\end{array}$ & $1530^{\mathrm{e}}$ & 1530 & 1984 & 0.819 & 2.0 & 0.3 & $\operatorname{tr}$ & 0.1 & - & $\begin{array}{c}\text { RI, MS, } \\
{ }^{13} \text { C-NMR }\end{array}$ \\
\hline 70 & $(E)-\alpha$-Bisabolene & 1530 & 1531 & 1761 & 0.751 & 0.6 & $\operatorname{tr}$ & - & - & - & $\begin{array}{c}\text { RI, MS, } \\
{ }^{13} \text { C-NMR }\end{array}$ \\
\hline 71 & $\begin{array}{c}(10 \beta \mathrm{H})-1 \beta, 8 \beta- \\
\text { Oxido-cadin-4-ene }\end{array}$ & $1534^{\mathrm{f}}$ & 1534 & 1853 & 0.830 & 9.3 & 0.4 & 7.3 & 1.3 & - & $\begin{array}{c}\text { RI, MS, } \\
{ }^{13} \mathrm{C}-\mathrm{NMR}\end{array}$ \\
\hline 72 & $\beta$-Elemol & 1541 & 1536 & 2077 & 0.819 & 0.4 & 0.7 & $\operatorname{tr}$ & 1.2 & 1.7 & $\begin{array}{l}\text { RI, MS, } \\
{ }^{13} \text { C-NMR }\end{array}$ \\
\hline 73 & (E)-Nerolidol & 1553 & 1547 & 2034 & 0.819 & 1.1 & 0.1 & 0.5 & 0.3 & - & $\begin{array}{c}\text { RI, MS, } \\
{ }^{13} \text { C-NMR }\end{array}$ \\
\hline 74 & Germacrene B \# & 1552 & 1549 & 1818 & 0.751 & - & 20.8 & 0.4 & 0.1 & 2.5 & $\begin{array}{l}\text { RI, MS, } \\
{ }^{13} \text { C-NMR }\end{array}$ \\
\hline 75 & Santalenone & 1576 & 1560 & 1980 & 0.841 & 13.0 & $\operatorname{tr}$ & - & - & - & $\begin{array}{c}\text { RI, MS, } \\
{ }^{13} \text { C-NMR }\end{array}$ \\
\hline 76 & $\begin{array}{l}\text { cis-Sesquisabinene } \\
\text { hydrate }\end{array}$ & $1565^{\mathrm{e}}$ & 1562 & 2079 & 0.819 & 0.8 & $\operatorname{tr}$ & 0.3 & 0.1 & - & $\begin{array}{c}\text { RI, MS, } \\
{ }^{13} \text { C-NMR }\end{array}$ \\
\hline 77 & $\begin{array}{l}\text { Germacra-1(10),5- } \\
\text { dien- } 4 \beta \text {-ol }\end{array}$ & $1572 \mathrm{~g}$ & 1564 & 2047 & 0.819 & 0.1 & $\operatorname{tr}$ & $\operatorname{tr}$ & 0.1 & - & $\begin{array}{l}\text { RI, MS, } \\
{ }^{13} C-N M R\end{array}$ \\
\hline 78 & Caryophyllene oxide & 1578 & 1567 & 1973 & 0.830 & 0.3 & 0.8 & 0.1 & - & 0.1 & $\begin{array}{c}\text { RI, MS, } \\
{ }^{13} \text { C-NMR }\end{array}$ \\
\hline 79 & Guaiol & 1593 & 1581 & 2119 & 0.819 & 2.3 & $\operatorname{tr}$ & $\operatorname{tr}$ & - & $\operatorname{tr}$ & $\begin{array}{c}\text { RI, MS, } \\
{ }^{13} \text { C-NMR }\end{array}$ \\
\hline 80 & Germacrene D-8-one & $1588^{h}$ & 1584 & 2066 & 0.841 & 0.5 & 0.1 & 8.7 & 0.6 & $\operatorname{tr}$ & $\begin{array}{c}\text { RI, MS, } \\
{ }^{13} \text { C-NMR }\end{array}$ \\
\hline 81 & Humulene oxide II & 1602 & 1597 & 2042 & 0.830 & 0.1 & $\operatorname{tr}$ & 0.4 & - & - & $\begin{array}{c}\text { RI, MS, } \\
{ }^{13} \text { C-NMR }\end{array}$ \\
\hline 82 & epi-Cubenol & 1602 & 1605 & 2046 & 0.819 & 0.2 & 0.3 & - & - & - & $\begin{array}{c}\text { RI, MS, } \\
{ }^{13} C-N M R\end{array}$ \\
\hline 83 & Alismol & 1619 & 1609 & 2245 & 0.830 & - & 0.6 & 0.1 & - & - & $\begin{array}{c}\text { RI, MS, } \\
{ }^{13} C-N M R\end{array}$ \\
\hline 84 & Eremoligenol & 1614 & 1614 & 2196 & 0.819 & 0.3 & - & - & - & 0.5 & $\begin{array}{c}\text { RI, MS, } \\
{ }^{13} \text { C-NMR }\end{array}$ \\
\hline 85 & $\gamma$-Eudesmol & 1618 & 1620 & 2172 & 0.819 & $\operatorname{tr}$ & 0.9 & 1.2 & $\operatorname{tr}$ & 0.4 & $\begin{array}{c}\text { RI, MS, } \\
{ }^{13} \text { C-NMR }\end{array}$ \\
\hline 86 & $\begin{array}{l}\text { Muurola-4,10(14)- } \\
\text { dien-8 } 8 \text {-ol }\end{array}$ & $1632^{\mathrm{i}}$ & 1629 & 2186 & 0.830 & 0.9 & 0.1 & 3.2 & 0.3 & - & $\begin{array}{l}\text { RI, MS, } \\
{ }^{13} \text { C-NMR }\end{array}$ \\
\hline 87 & $\beta$-Eudesmol & 1641 & 1635 & 2225 & 0.819 & $\operatorname{tr}$ & 0.2 & - & - & 22.5 & $\begin{array}{c}\text { RI, MS, } \\
{ }^{13} \text { C-NMR }\end{array}$ \\
\hline 88 & $\alpha$-Cadinol & 1643 & 1637 & 2231 & 0.819 & 0.2 & 0.7 & 0.6 & $\operatorname{tr}$ & - & $\begin{array}{c}\text { RI, MS, } \\
{ }^{13} \text { C-NMR }\end{array}$ \\
\hline
\end{tabular}


Table 1. Cont.

\begin{tabular}{|c|c|c|c|c|c|c|c|c|c|c|c|}
\hline $\mathbf{N}^{\circ}$ & Compounds $^{a}$ & $\mathrm{RIl}^{\mathrm{b}}$ & RIa & RIp & RRF & S2 (\%) & S14 (\%) & S24 (\%) & S44 (\%) & S47 (\%) & Identification \\
\hline 89 & $\alpha$-Eudesmol & 1653 & 1639 & 2216 & 0.819 & - & 0.1 & - & - & 12.4 & $\begin{array}{c}\text { RI, MS, } \\
{ }^{13} \text { C-NMR }\end{array}$ \\
\hline 90 & Tumerone-ar & 1643 & 1642 & 2252 & 0.841 & - & - & 0.1 & - & 0.1 & RI, MS \\
\hline 91 & $\beta$-Bisabolol & 1659 & 1653 & 2144 & 0.819 & 1.1 & 0.1 & 0.2 & $\operatorname{tr}$ & - & $\begin{array}{c}\text { RI, MS, } \\
{ }^{13} \text { C-NMR }\end{array}$ \\
\hline 92 & $\begin{array}{c}(7 \alpha \mathrm{H}) \text {-Germacrene } \\
\text { D- } 8 \alpha-\mathrm{ol}^{*}\end{array}$ & $1657^{f}$ & 1657 & 2355 & 0.819 & $\operatorname{tr}$ & - & 7.8 & 0.7 & - & $\begin{array}{c}\text { RI, MS, } \\
{ }^{13} \text { C-NMR }\end{array}$ \\
\hline 93 & $\begin{array}{c}\text { (7 } \alpha \mathrm{H}) \text {-Germacrene } \\
\text { D- } 8 \beta-\mathrm{ol} \text { * }\end{array}$ & $1657^{\mathrm{f}}$ & 1657 & 2355 & 0.819 & $\operatorname{tr}$ & - & 2.6 & 0.2 & - & $\begin{array}{c}\text { RI, MS, } \\
{ }^{13} \text { C-NMR }\end{array}$ \\
\hline 94 & $\alpha$-Bisabolol & 1673 & 1664 & 2208 & 0.819 & 0.7 & $\operatorname{tr}$ & 1.4 & 0.1 & - & $\begin{array}{c}\text { RI, MS, } \\
{ }^{13} \text { C-NMR }\end{array}$ \\
\hline 95 & epi- $\alpha$-Bisabolol & $1667^{j}$ & 1667 & 2214 & 0.819 & 0.7 & $\operatorname{tr}$ & 0.1 & - & - & $\begin{array}{c}\text { RI, MS, } \\
{ }^{13} \text { C-NMR }\end{array}$ \\
\hline 96 & $\begin{array}{c}\text { Cadina-1(10),4-dien- } \\
8 \beta \text {-ol }\end{array}$ & $1676^{f}$ & 1676 & 2276 & 0.819 & $\operatorname{tr}$ & 0.3 & 7.6 & 0.3 & - & $\begin{array}{c}\text { RI, MS, } \\
{ }^{13} \text { C-NMR }\end{array}$ \\
\hline 97 & $\begin{array}{l}\text { Cadina-4,10(14)- } \\
\quad \text { dien-8 } 8 \text {-ol } \\
(1 \beta H, 5 \beta H)-6,12-\end{array}$ & $1675^{\mathrm{i}}$ & 1678 & 2280 & 0.830 & 0.1 & 0.4 & 0.8 & - & - & $\begin{array}{c}\text { RI, MS, } \\
{ }^{13} \text { C-NMR }\end{array}$ \\
\hline 98 & $\begin{array}{c}\text { Oxido-guaia- } \\
6,10(14), 11(12) \text {-trien- } \\
4 \alpha \text {-ol }\end{array}$ & $1754^{\mathrm{c}}$ & 1754 & 2519 & 0.853 & $\operatorname{tr}$ & 1.7 & - & - & 0.1 & $\begin{array}{c}\text { RI, MS, } \\
{ }^{13} \text { C-NMR }\end{array}$ \\
\hline \multirow[t]{7}{*}{99} & $\begin{array}{c}(6 \alpha \mathrm{H}) \text {-Germacra- } \\
1(10), 4,7(11)- \\
\text { trien-6,12- } \gamma \text {-lactone }\end{array}$ & $1856^{c}$ & 1856 & 2829 & 0.985 & - & 0.8 & - & - & - & $\begin{array}{c}\text { RI, MS, } \\
{ }^{13} \text { C-NMR }\end{array}$ \\
\hline & $\begin{array}{l}\text { Hydrocarbon } \\
\text { monoterpenes }\end{array}$ & & & & & 3.2 & 19.9 & 10.0 & 70.9 & 15.7 & \\
\hline & $\begin{array}{l}\text { Oxygenated } \\
\text { monoterpenes }\end{array}$ & & & & & 2.1 & 0.5 & 0.2 & 0.3 & 1.3 & \\
\hline & $\begin{array}{l}\text { Hydrocarbon } \\
\text { sesquiterpenes }\end{array}$ & & & & & 58.1 & 56.1 & 39.6 & 22.2 & 40.9 & \\
\hline & $\begin{array}{c}\text { Oxygenated } \\
\text { sesquiterpenes }\end{array}$ & & & & & 34.4 & 19.9 & 44.0 & 5.5 & 39.8 & \\
\hline & Other compounds & & & & & - & - & - & - & 0.4 & \\
\hline & Total & & & & & 97.8 & 96.4 & 93.8 & 98.9 & 98.1 & \\
\hline
\end{tabular}

${ }^{a}$ Order of elution and percentages are given on an apolar column (BP-1), except components with an asterisk $\left(^{*}\right)$, where percentages are taken on a polar column (BP-20). (\#) Thermolabile compound, percentage evaluated by a combination of GC-FID and ${ }^{13} \mathrm{C}-\mathrm{NMR}$ data [23,24]. ${ }^{b}$ RIl: Retention indices reported in the Terpenoids Library Website [25] or in reference ${ }^{\mathrm{c}}$ [15]; ${ }^{\mathrm{d}}$ [26]; ${ }^{\mathrm{e}}[27] ;{ }^{\mathrm{f}}[16] ;{ }^{\mathrm{g}}$ [28]; ${ }^{\mathrm{h}}$ [14]]; ${ }^{\mathrm{i}}[29] ;$ j [30]. RIa, RIp: retention indices measured on apolar and polar capillary column, respectively. RRF: relative response factors calculated using methyl octanoate as internal standard. The relative proportions of constituent are expressed in g/ $100 \mathrm{~g}$. (-): not detected; tr: traces level $(<0.05 \%)$. S2: sample 2; the same for S14, S24, S44 and S47. ${ }^{13}$ C-NMR: compounds identified by NMR in the essential oil samples and obvious in at least one fraction of chromatography; ${ }^{13} C-N M R$ (italic): compounds identified by NMR in fractions of chromatography.

\subsection{Chemical Variability of Leaf Essential Oil from I. dewevrei}

The chemical variability of the leaf essential oil from I. dewevrei was evaluated throughout 47 samples collected from shrubs at different phenological stages, at six sites of the Bossématié forest (Eastern Côte d'Ivoire) and two sites of the Haut-Sassandra forest (Western Côte d'Ivoire), during the rainy and dry seasons. In total, 113 constituents accounting for $90.8-98.9 \%$ of the samples' whole compositions were identified (Supplementary Materials, Table S1). All the samples except one (46/47) were largely dominated by sesquiterpenes $(57.3-92.5 \%)$. Monoterpenes were prevalent in a single sample $(71.2 \%)$, and the main components varied drastically from sample to sample: $(Z)-\beta$-ocimene $(0.1-36.6 \%)$, germacrene $\mathrm{D}(0.2-34.0 \%)$, (E)- $\beta$-ocimene $(0-30.5 \%)$, germacrene B $(0.1-24.8 \%), \beta$-eudesmol $(0-22.5 \%)$, $(10 \beta \mathrm{H})-1 \beta, 8 \beta$-oxido-cadina-4-ene (0-13.7\%), $(5 \alpha \mathrm{H}, 10 \beta \mathrm{Me})-6,12$-oxido-elema-1,3,6,11(12)tetraene $(0-13.7 \%)$, limonene $(\operatorname{tr}-13.0 \%)$, santalenone $(0-13.0 \%),(E)$ - $\beta$-caryophyllene (1.7-12.4\%), $\alpha$-eudesmol (0-12.4\%), trans- $\alpha$-bergamotene $(0-11.7 \%)$, caryophyllene oxide $(0-11.5 \%)$ and trans- $\beta$-bergamotene (0-10.9\%). Therefore, the 47 essential oil compositions 
were subjected to a hierarchical cluster analysis (HCA) and principal component analysis (PCA) to highlight possible chemical variability.

The dendrogram from the HCA revealed three distinct groups within the 47 investigated oil samples: Group I (9 samples), Group II (19 samples) and Group III (19 samples), each consisting of two subgroups (Figure 1). The first principal factor of the PCA (F1: $44.31 \%$ ), the second (F2: $22.52 \%$ ) and the third (F3: $13.01 \%$ ) accounted for $79.12 \%$ of the total variance of the chemical composition. The PCA map of the samples' distribution relative to the principal axes F1 and F3 (57.32\%) confirmed the three chemical composition groups (Figure 2). The Groups II and III were more homogenous than Group I. The mean contents $(\mathrm{M})$ and the standard deviation (SD) of the majority compounds of the different subgroups are reported in Table 2.

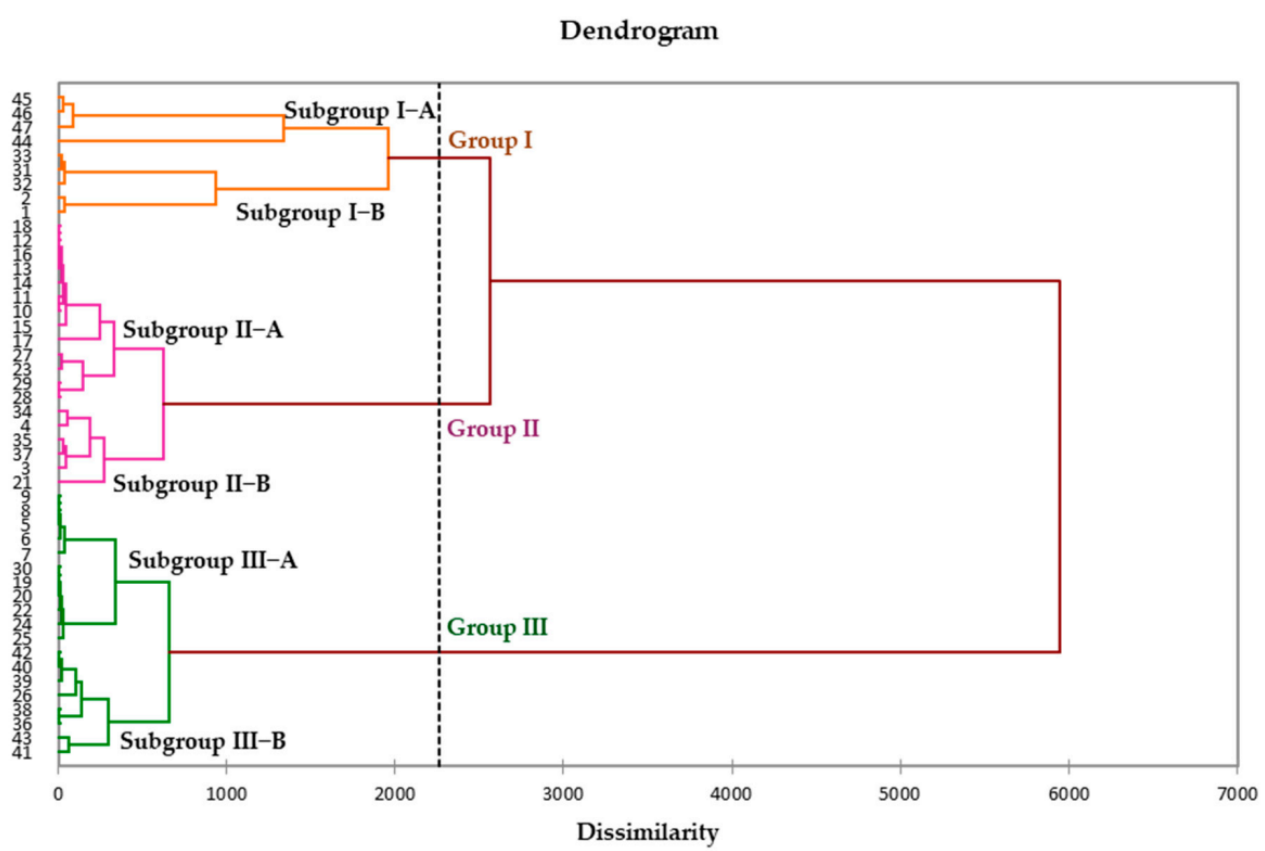

Figure 1. Dendrogram of hierarchical cluster analysis (HCA) of the 47 leaf oil samples from I. dewevrei.

Observations (axes F1 and F3 : $57.32 \%$ )

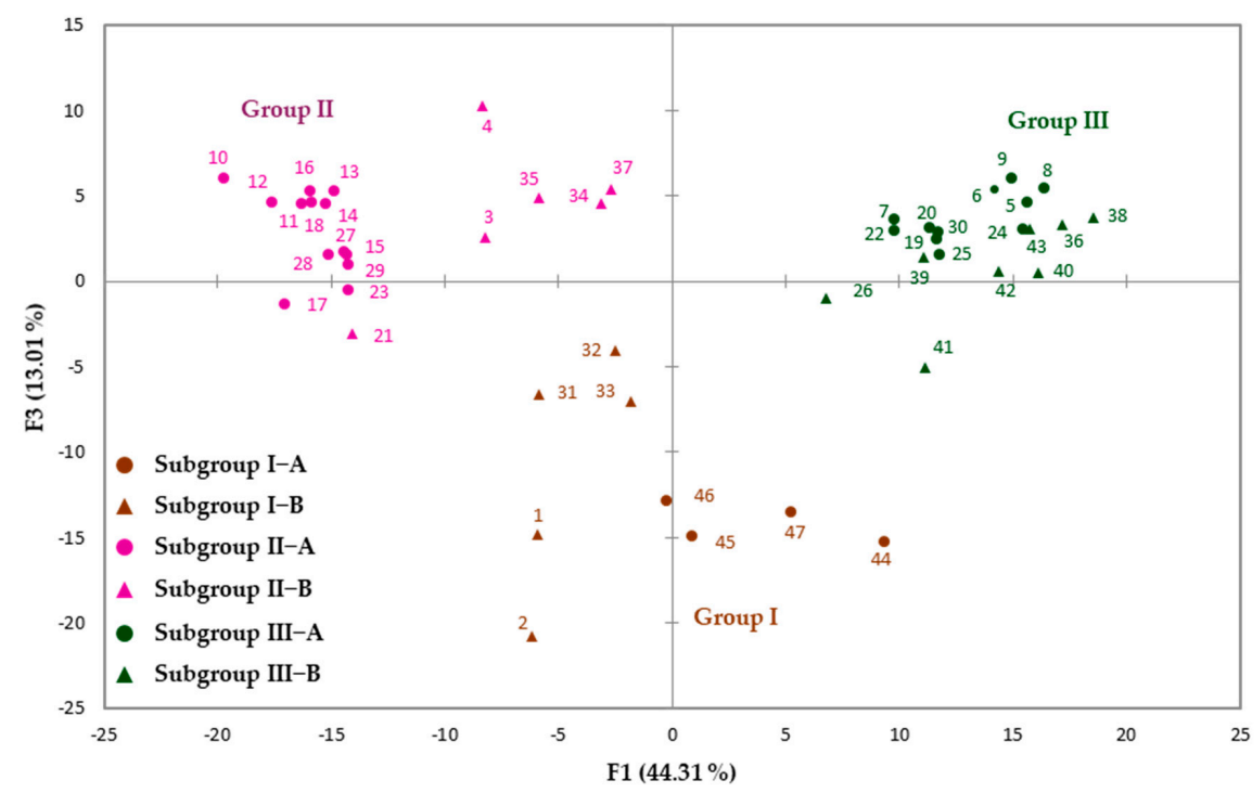

Figure 2. Principal component analysis (PCA) of the 47 leaf oil samples from I. dewevrei. 
Table 2. Chemical variability of the leaf essential oil from Isolona dewevrei (main constituents).

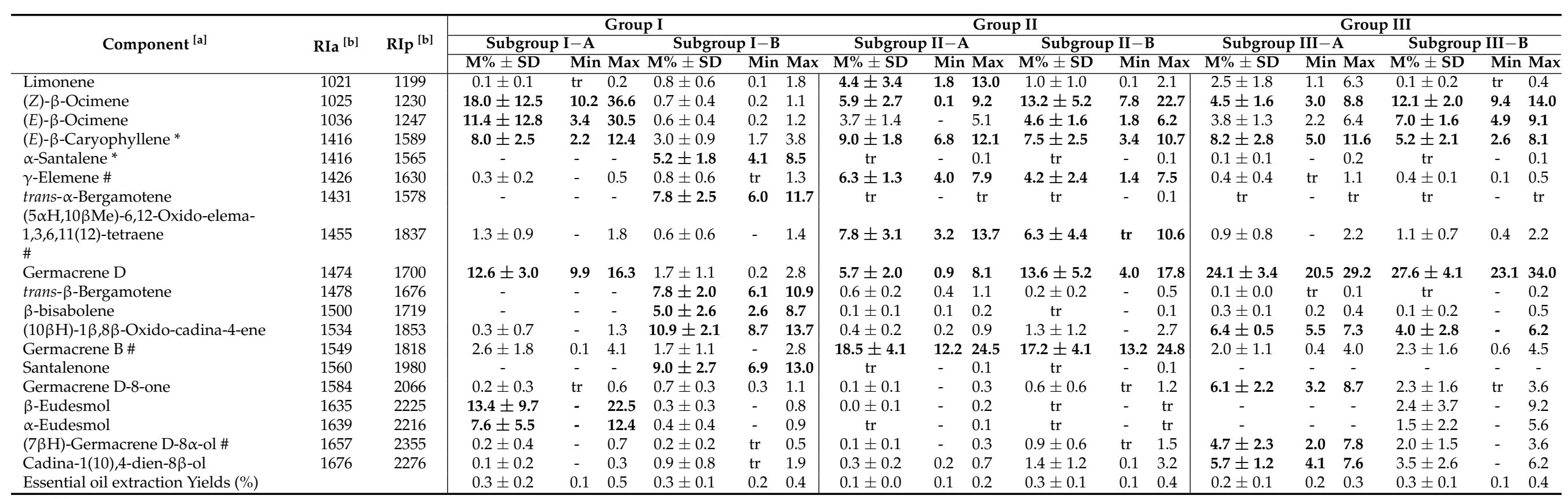

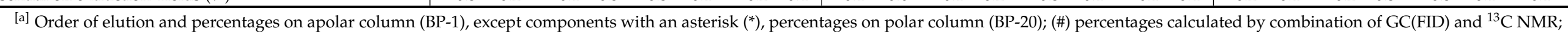

${ }^{[b]}$ RIa, RIp: Retention indices measured on apolar and polar capillary column, respectively; M\% \pm SD: mean percentage and standard deviation; (-): not detected; tr: traces level ( $\left.<0.05 \%\right)$. 
The Subgroup I-A (four oil samples) was dominated by $(Z)-\beta$-ocimene $(\mathrm{M}=18.0 \%$, $\mathrm{SD}=12.5 \%)$, $\beta$-eudesmol $(\mathrm{M}=13.4 \%, \mathrm{SD}=9.7 \%)$, germacrene $\mathrm{D}(\mathrm{M}=12.6 \%, \mathrm{SD}=3.0 \%)$, $(E)$ - $\beta$-ocimene $(\mathrm{M}=11.4 \%, \mathrm{SD}=12.8 \%),(E)$ - $\beta$-caryophyllene $(\mathrm{M}=8.0 \%, \mathrm{SD}=2.5 \%)$ and $\alpha$ eudesmol $(\mathrm{M}=7.6 \%, \mathrm{SD}=5.5 \%)$. The major compounds of Subgroup I-B (five oil samples) were $(10 \beta \mathrm{H})-1 \beta, 8 \beta$-oxido-cadina-4-ene $(\mathrm{M}=10.9 \%, \mathrm{SD}=2.1 \%)$, santalenone $(\mathrm{M}=9.0 \%$, $\mathrm{SD}=2.7 \%)$, trans- $\alpha$-bergamotene $(\mathrm{M}=7.8 \%, \mathrm{SD}=2.5 \%)$, trans- $\beta$-bergamotene $(\mathrm{M}=7.8 \%$, $\mathrm{SD}=2.0 \%), \alpha$-santalene $(\mathrm{M}=5.2 \%, \mathrm{SD}=1.8 \%)$ and $\beta$-bisabolene $(\mathrm{M}=5.0 \%, \mathrm{SD}=2.6 \%)$. Santalenone, trans- $\alpha$-bergamotene, trans- $\beta$-bergamotene, $\alpha$-santalene and $\beta$-bisabolene, present in Subgroup I-B, were absent from Subgroup I-A and did not exceed 1.1\% in Groups II and III. $\beta$-eudesmol and $\alpha$-eudesmol did not exceed $0.2 \%$ in Group II and were absent from Subgroup III-A.

The Group II is characterized by its high content of germacrene $\mathrm{B},(5 \alpha \mathrm{H}, 10 \beta \mathrm{Me})$ 6,12-oxido-elema-1,3,6,11(12)-tetraene, $\gamma$-elemene and limonene. The main constituents of Subgroup II-A, which consisted of 13 oil samples, were germacrene B $(\mathrm{M}=18.5 \%$, $\mathrm{SD}=4.1 \%),(E)-\beta$-caryophyllene $(\mathrm{M}=9.0 \%, \mathrm{SD}=1.8 \%),(5 \alpha \mathrm{H}, 10 \beta \mathrm{Me})-6,12$-oxido-elema1,3,6,11(12)-tetraene $(\mathrm{M}=7.8 \%, \mathrm{SD}=3.1 \%), \gamma$-elemene $(\mathrm{M}=6.3 \%, \mathrm{SD}=1.3 \%),(Z)$ - $\beta$-ocimene $(\mathrm{M}=5.9 \%, \mathrm{SD}=2.7 \%)$, germacrene $\mathrm{D}(\mathrm{M}=5.7 \%, \mathrm{SD}=2.0 \%)$ and limonene $(\mathrm{M}=4.4 \%$, $\mathrm{SD}=3.4 \%$ ). The Subgroup II-B (six oil samples) was also dominated by germacrene $\mathrm{B}(\mathrm{M}=17.2 \%, \mathrm{SD}=4.1 \%)$, followed by germacrene $\mathrm{D}(\mathrm{M}=13.6 \%, \mathrm{SD}=5.2 \%),(Z)-\beta-$ ocimene $(\mathrm{M}=13.2 \%, \mathrm{SD}=5.2 \%),(E)$ - $\beta$-caryophyllene $(\mathrm{M}=7.5 \%, \mathrm{SD}=2.5 \%),(5 \alpha \mathrm{H}, 10 \beta \mathrm{Me})$ 6,12-oxido-elema-1,3,6,11(12)-tetraene $(\mathrm{M}=6.3 \%$, $\mathrm{SD}=4.4 \%),(E)$ - $\beta$-ocimene $(\mathrm{M}=4.6 \%$, $\mathrm{SD}=1.6 \%)$ and $\gamma$-elemene $(\mathrm{M}=4.2 \%, \mathrm{SD}=2.4 \%)$.

Group III differed from Groups I and II by its important proportions of germacrene D-8one, $(7 \beta \mathrm{H})$-germacrene D-8 $\alpha$-ol and cadina-1(10),4-dien-8$\beta$-ol. The Subgroup III-A (11 oil samples) displayed germacrene $\mathrm{D}(\mathrm{M}=24.1 \%, \mathrm{SD}=3.4 \%),(E)-\beta$-caryophyllene $(\mathrm{M}=8.2 \%$, $\mathrm{SD}=2.8 \%),(10 \beta \mathrm{H})-1 \beta, 8 \beta$-oxido-cadina-4-ene $(\mathrm{M}=6.4 \%, \mathrm{SD}=0.5 \%)$, germacrene $\mathrm{D}$-8-one $(\mathrm{M}=6.1 \%, \mathrm{SD}=2.2 \%)$, cadina-1(10),4-dien-8 $\beta$-ol $(\mathrm{M}=5.7 \%, \mathrm{SD}=1.2 \%),(7 \beta \mathrm{H})$-germacrene $\mathrm{D}-8$ - $\alpha$-ol $(\mathrm{M}=4.7 \%, \mathrm{SD}=2.3 \%)$ and $(Z)-\beta$-ocimene $(\mathrm{M}=4.5 \%, \mathrm{SD}=1.6 \%)$ as the most prevalent compounds. Finally, germacrene $\mathrm{D}(\mathrm{M}=27.6 \%, \mathrm{SD}=4.1 \%)$, followed this time by $(Z)$ - $\beta$-ocimene $(\mathrm{M}=12.1 \%, \mathrm{SD}=2.0 \%),(E)$ - $\beta$-ocimene $(\mathrm{M}=7.0 \%, \mathrm{SD}=1.6 \%),(E)-\beta$ caryophyllene $(\mathrm{M}=5.2 \%, \mathrm{SD}=2.1 \%)$ and $(10 \beta \mathrm{H})-1 \beta, 8 \beta$-oxido-cadina-4-ene $(\mathrm{M}=4.0 \%$, $\mathrm{SD}=2.8 \%$ ), were the majority components of Subgroup III-B (eight oil samples).

This study demonstrated both qualitative and quantitative variations of constituents within the detected groups and subgroups. The observed chemical variability of the leaf essential oil from $I$. dewevrei was probably due to several external factors, although genetic factors could not be completely excluded. As all samples were collected from shrubs at different phenological stages, at six sites of the Bossématié forest (Sites 1 to 6; Eastern Côte d'Ivoire) and two sites of the Haut-Sassandra forest (Sites 7 and 8; Western Côte d'Ivoire), during the rainy and dry seasons, the effect of phenology, season and harvest site on samples' distribution into groups was examined (Figure 3). Regarding the phenology, samples collected from shrubs bearing flowers are all included in Group I, where they constituted the Subgroup I-A. Moreover, Group II is exclusively constituted of samples collected from shrubs bearing neither flowers nor fruits, while all the samples collected from shrubs bearing fruits are included in Group III. Therefore, the phenology appeared to be an important factor explaining the chemical variability of the leaf essential oil of the plant. From a seasonal point of view, samples collected during the dry season are more differenciated than those harvested during the rainy season. Indeed, the three barycenters of the samples collected during the dry season, corresponding to the three chemical groups, are furthest from the origin. In addition, Subgroups I-B, II-B and III-B exclusively consisted of samples collected during the rainy season, whereas samples from Subgroup I-A were harvested during the dry season. The effect of seasons on the chemical variability of $I$. dewevrei leaf oil could not therefore be overlooked. Although all the harvest sites are located in the same climatic zone (mesophilic sector; Celtis spp., Triplochiton scleroxylon and its variant of Nesogordonia papaverifera and Khaya ivorensis forests) with the same soil 
type (ferrallitic), the sites' effect on the chemical variability was noticeable. In fact, the Subgroup I-A consisted of samples from Site 8, while those collected at Sites 3 and 4 were all included in Subgroup II-A. Samples from Site 7 are all included in Subgroup III-B, whereas those from Site 2 belonged to Group III. The harvest sites being sufficiently distant, their effect on the chemical variability could be related to possible microclimates or genetic differences. It could finally be argued that phenology, season and harvest site really impacted the chemical variability of the leaf essential oil from I. dewevrei.

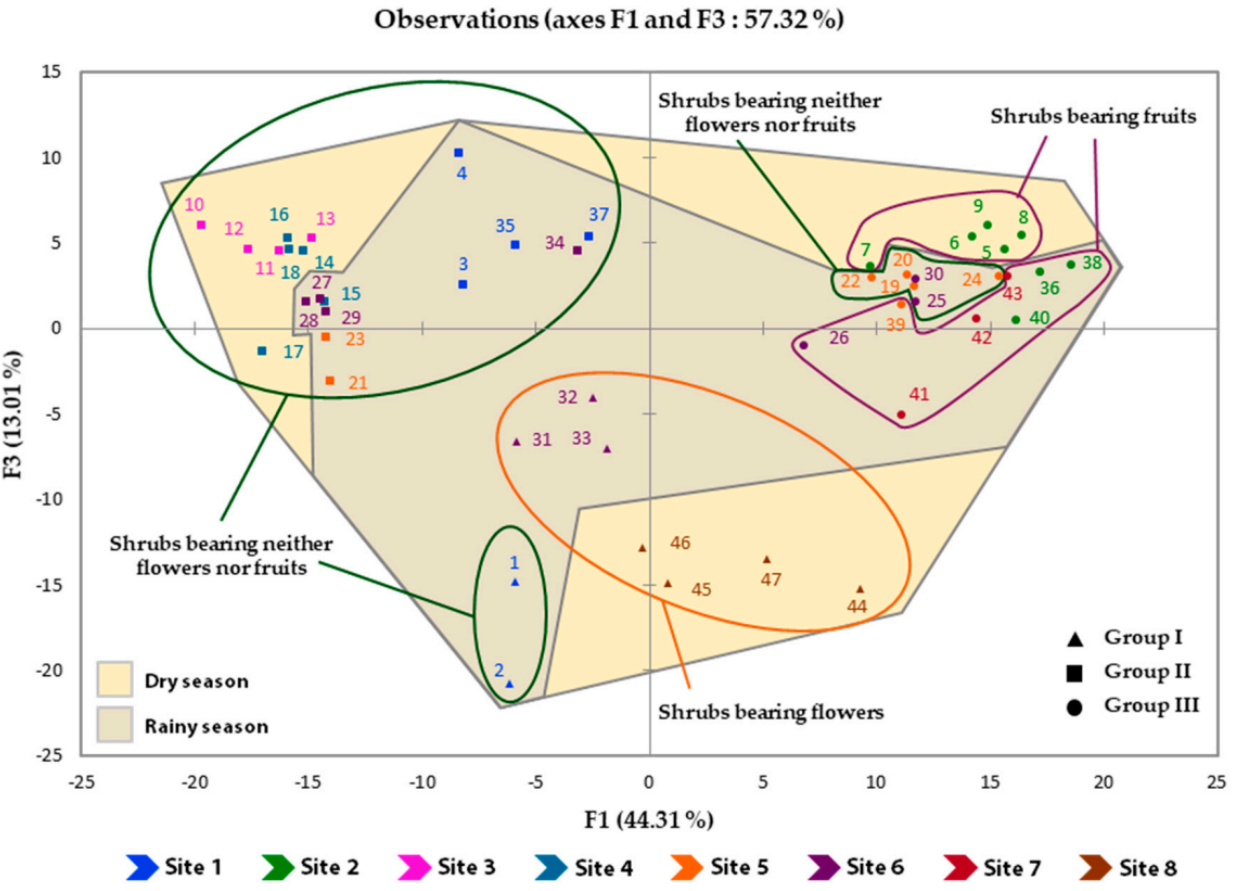

Figure 3. Effect of phenology, season and harvest site on the 47 oil samples' distribution into groups.

\subsection{Comparision to Literature Data}

The chemical composition of the leaf essential oil from $I$. dewevrei differed considerably from that from I. cooperi and I. campanulata. Indeed, unlike leaves of I. dewevrei which produced a sesquiterpene-rich oil, the leaf oil from $I$. cooperi was largely dominated by monoterpenes: (Z)- $\beta$-ocimene $(16.9-71.1 \%), \gamma$-terpinene $(0.6-26.3 \%), \alpha$-terpinene $(0.3-12.7 \%), \delta$ 3 -carene (up to $8.8 \%$ ), 5[(Z)-hexylidene]-5H-furan-2-one (1.2-7.8\%) and massoia lactone $(0.9-4.2 \%)$ [12]. Only $(Z)-\beta$-ocimene $(0.1-26.7 \%)$ was a major compound of $I$. dewevrei samples, while $\gamma$-terpinene, $\alpha$-terpinene and $\delta$-3-carene did not exceed $1.1 \%$. Moreover, $5[(Z)$ hexylidene]-5H-furan-2-one and massoia lactone were not detected in $I$. dewevrei samples. The leaf oil from I. campanulata, on the other hand, was dominated by $(E)-\beta$-caryophyllene (8.2-30.5\%), eudesm-5-en-11-ol (0.1-20.0\%) and $\alpha$-humulene (3.8-17.5\%) [13]. Although (E)- $\beta$-caryophyllene (1.7-12.4\%) was predominant in some $I$. dewevrei samples, its contents remained lower. In addition, eudesm-5-en-11-ol was completely absent from the leaf oil of I. dewevrei, while $\alpha$-humulene was present in relatively small proportions (0.9-2.9\%). Most of the other major constituents of $I$. dewevrei leaf oil were either absent or present at low contents in I. cooperi and I. campanulata leaf essential oils.

\subsection{Evaluation of In Vitro Anti-Inflammatory Activity}

The in vitro anti-inflammatory potential of $I$. dewevrei leaf essential oil (S44) was evaluated by determining its ability to inhibit lipoxygenases (LOX). Indeed, LOXs are nonheme iron-containing dioxygenases that convert linoleic, arachidonic and other polyunsaturated fatty acid into biologically active metabolites involved in the inflammatory and immune responses. Several inflammatory processes such as arthritis, bronchial asthma and cancer are associated with an important production of leukotrienes catalyzed by the LOX path- 
way from arachidonic acid [31-34]. The inhibition of the LOX pathway with inhibitors of LOX would prevent the production of leukotrienes and therefore could constitute a therapeutic target for the treating of human inflammation-related diseases. Thus, the search for new LOX inhibitors appears as critical because many exhibit significant in vitro anti-inflammatory activity.

The ability of $I$. dewevrei leaf essential oil to inhibit soybean lipoxygenase was determined as an indication of potential in vitro anti-inflammatory activity. I. dewevrei leaf essential oil exhibited an inhibition of LOX activity (Table 3). The percentage of inhibition increases with the concentration of the oil i.e., $10.3 \%$ at $0.005 \mathrm{mg} / \mathrm{mL}$ to $51.5 \%$ at $0.020 \mathrm{mg} / \mathrm{mL}$ of the essential oil. The $\mathrm{IC}_{50}$ values (concentration at which $50 \%$ of the lipoxygenase was inhibited) were determined for the I. dewevrei leaf essential oil and for the non-competitive inhibitor of lipoxygenase, the nordihydroguaiaretic acid (NDGA) (Table 3), usually used as a reference in in vitro anti-inflammatory assays [32-34]. Data showed that the $\mathrm{IC}_{50}$ value of I. dewevrei leaf essential oil $(0.020 \pm 0.005 \mathrm{mg} / \mathrm{mL})$ is slightly higher than the $\mathrm{IC}_{50}$ value of NDGA $(0.013 \pm 0.003 \mathrm{mg} / \mathrm{mL})$. The low ratio between the two values of $\mathrm{IC}_{50}$ (I. dewevrei leaf essential oil vs. NDGA) makes it possible to consider this essential oil as a high inhibitor of the LOX activity, suggesting an in vitro anti-inflammatory potential [35].

Table 3. $\mathrm{IC}_{50}$ values and in vitro anti-inflammatory activity of Isolona dewevrei leaf essential oil.

\begin{tabular}{cccccc}
\hline & \multicolumn{2}{c}{ Percentage Inhibition of LOX } & \multicolumn{2}{c}{ IC $_{\mathbf{5 0}}(\mathbf{m g} / \mathbf{m L})$} \\
\hline Concentration \# & Inhibition (\%) & Concentration \# & Inhibition (\%) & Essential oil & $0.020 \pm 0.005$ \\
0.005 & $10.3 \pm 0.8$ & 0.015 & $47.9 \pm 10.4$ & $*$ NDGA & $0.013 \pm 0.003$ \\
0.010 & $12.7 \pm 0.7$ & 0.020 & $51.5 \pm 13.8$ & & \\
\hline
\end{tabular}

Values are means of triplicates \pm standard deviation; ${ }^{*}$ NDGA: NorDihydroGuaiaretic Acid; $\# \mathrm{mg} / \mathrm{mL}$.

\section{Material and Methods}

\subsection{Plant Material}

The fresh leaves' samples were collected on individual I. dewevrei shrubs at different phenological stages, at six sufficiently distant sites (Sites 1 to 6) of the Bossématié forest, Region of Abengourou, Eastern Côte d'Ivoire, and at two sites (Sites 7 and 8) of the Haut-Sassandra forest, Western Côte d'Ivoire. Geographical coordinates: Site 1 $\left(6^{\circ} 31^{\prime} 34.7^{\prime \prime} \mathrm{N}\right.$ and $\left.3^{\circ} 28^{\prime} 15.0^{\prime \prime} \mathrm{W}\right)$, Site $2\left(6^{\circ} 29^{\prime} 26.0^{\prime \prime} \mathrm{N}\right.$ and $\left.3^{\circ} 29^{\prime} 11.7^{\prime \prime} \mathrm{W}\right)$, Site $3\left(6^{\circ} 27^{\prime} 44.9^{\prime \prime}\right.$ $\mathrm{N}$ and $\left.3^{\circ} 32^{\prime} 25.5^{\prime \prime} \mathrm{W}\right)$, Site $4\left(6^{\circ} 25^{\prime} 44.5^{\prime \prime} \mathrm{N}\right.$ and $\left.3^{\circ} 32^{\prime} 40.7^{\prime \prime} \mathrm{W}\right)$, Site $5\left(6^{\circ} 26^{\prime} 07.2^{\prime \prime} \mathrm{N}\right.$ and $\left.3^{\circ} 28^{\prime} 27.2^{\prime \prime} \mathrm{W}\right)$, Site $6\left(6^{\circ} 23^{\prime} 43.4^{\prime \prime} \mathrm{N}\right.$ and $\left.3^{\circ} 25^{\prime} 59.4^{\prime \prime} \mathrm{W}\right)$, Site $7\left(6^{\circ} 53^{\prime} 40.2^{\prime \prime} \mathrm{N}\right.$ and $\left.6^{\circ} 55^{\prime} 36.3^{\prime \prime} \mathrm{W}\right)$ and Site $8\left(6^{\circ} 54^{\prime} 52.7^{\prime \prime} \mathrm{N}\right.$ and $\left.6^{\circ} 57^{\prime} 21.1^{\prime \prime} \mathrm{W}\right)$. The harvest took place on one hand during the dry season (March 2016, January 2017 and February 2021) and during the rainy season on the other hand (April 2016, August 2016 and July 2020) (Supplementary Materials, Table S2). Plant material was authenticated by botanists from the Centre Suisse de Recherches Scientifiques (CSRS) and Centre National de Floristique (CNF) Abidjan, Côte d'Ivoire. A voucher specimen was deposited at the herbarium of CNF, Abidjan, with the reference LAA 12874.

\subsection{Essential Oil Isolation and Fractionation}

The essential oil samples were obtained by the hydrodistillation of fresh leaves for $3 \mathrm{~h}$ each, using a Clevenger-type apparatus. Yields were calculated from fresh material $(w / w)$. Plant material and essential oil extraction data are reported in Table S2 (Supplementary material). The leaf essential oil samples S2 (4.115 g), S14 (4.820 g), S24 (3.702 g), S44 (4.226 g) and S47 (3.910 g) were separately chromatographed on a column with silica gel (Acros Organics, Waltham, MA, USA, 60-200 $\mu \mathrm{m}, 120 \mathrm{~g}$ each, except S14, $150 \mathrm{~g}$ ), using a gradient of solvents, distilled $n$-pentane (VWR Chemicals, Radnor, PA, USA, 99\%)/diethyl ether (VWR Chemicals, $100.0 \%$ ) of increasing polarity (P/DE, 100/0 to 0/100). Seven fractions were eluted for each oil sample: F1 and F2 (eluted with $n$-pentane) contained hydrocarbons; 
F3-F6 (eluted with P/DE mixtures) contained medium polar compounds; F7 (eluted with diethyl ether) contained polar compounds. The respective weights of the fractions from the different samples are reported in the Table 4.

Table 4. Weights of fractions from column chromatography.

\begin{tabular}{|c|c|c|c|c|c|c|c|c|}
\hline \multirow[b]{2}{*}{ Samples } & \multicolumn{7}{|c|}{ Fractions Weights (g) } & \multirow[b]{2}{*}{ Total (g) } \\
\hline & $\begin{array}{c}\text { F1 } \\
\text { P: } 100 \%\end{array}$ & $\begin{array}{c}\text { F2 } \\
\text { P: } 100 \%\end{array}$ & $\begin{array}{c}\text { F3 } \\
\text { P/DE: } 98 / 2 \%\end{array}$ & $\begin{array}{c}\text { F4 } \\
\text { P/DE: } 95 / 5 \%\end{array}$ & $\begin{array}{c}\text { F5 } \\
\text { P/DE: } 90 / 10 \%\end{array}$ & $\begin{array}{c}\text { F6 } \\
\text { P/DE: } 80 / 20 \%\end{array}$ & $\begin{array}{c}\text { F7 } \\
\text { DE: } 100 \%\end{array}$ & \\
\hline S2 (4.115 g) & 1.301 & 1.187 & 0.197 & 0.402 & 0.489 & 0.212 & 0.307 & 4.095 \\
\hline S14 (4.820 g) & 2.470 & 1.109 & 0.144 & 0.285 & 0.413 & 0.141 & 0.190 & 4.752 \\
\hline S24 (3.702 g) & 1.221 & 0.506 & 0.201 & 0.478 & 0.615 & 0.246 & 0.352 & 3.619 \\
\hline S44 (4.226 g) & 2.543 & 1.371 & 0.031 & 0.089 & 0.082 & 0.029 & 0.032 & 4.177 \\
\hline S47 (3.910 g) & 1.256 & 0.940 & 0.184 & 0.471 & 0.521 & 0.179 & 0.312 & 3.863 \\
\hline
\end{tabular}

P: $n$-pentane; DE: Diethyl ether.

\subsection{Gas Chromatography}

Analyses were performed on a Clarus 500 PerkinElmer Chromatograph (PerkinElmer, Courtaboeuf, France), equipped with a flame ionization detector (FID) and two fused-silica capillary columns ( $50 \mathrm{~m} \times 0.22 \mathrm{~mm}$, film thickness $0.25 \mu \mathrm{m}$ ), BP-1 (polydimethylsiloxane) and BP-20 (polyethylene glycol). The oven temperature was programmed from $60{ }^{\circ} \mathrm{C}$ to $220^{\circ} \mathrm{C}$ at $2{ }^{\circ} \mathrm{C} / \mathrm{min}$ and then held isothermal at $220^{\circ} \mathrm{C}$ for $20 \mathrm{~min}$; injector temperature: $250{ }^{\circ} \mathrm{C}$; detector temperature: $250{ }^{\circ} \mathrm{C}$; carrier gas: hydrogen $(0.8 \mathrm{~mL} / \mathrm{min})$; split: $1 / 60$; injected volume: $0.5 \mu \mathrm{L}$. Retention indices (RI) were determined relative to the retention times of a series of n-alkanes (C8-C29) with a linear interpolation (« Target Compounds » software from PerkinElmer). The relative response factor (RFF) of each compound was calculated according to the International Organization of the Flavor Industry (IOFI)recommended practice for the use of predicted relative response factors for the rapid quantification of volatile flavoring compounds by GC(FID) [36]. Methyl octanoate was used as an internal reference, and the relative proportion of each constituent (expressed in $\mathrm{g} / 100 \mathrm{~g}$ ) was calculated using the weight of the essential oil and reference, peak area and relative response factors (RRF).

\subsection{Gas Chromatography-Mass Spectrometry in Electron Impact Mode}

The essential oil samples and all fractions of chromatography were analyzed with a Clarus SQ8S PerkinElmer TurboMass detector (quadrupole), directly coupled with a Clarus 580 PerkinElmer Autosystem XL (PerkinElmer, Courtaboeuf, France), equipped with a Rtx-1 (polydimethylsiloxane) fused-silica capillary column $(60 \mathrm{~m} \times 0.22 \mathrm{~mm}$ i.d., film thickness $0.25 \mu \mathrm{m}$ ). The oven temperature was programmed from 60 to $230^{\circ} \mathrm{C}$ at $2^{\circ} / \mathrm{min}$ and then held isothermal for $45 \mathrm{~min}$; injector temperature, $250^{\circ} \mathrm{C}$; ion-source temperature, $250^{\circ} \mathrm{C}$; carrier gas, $\mathrm{He}(1 \mathrm{~mL} / \mathrm{min})$; split ratio, 1:80; injection volume, $0.2 \mu \mathrm{L}$; ionization energy, $70 \mathrm{eV}$. The electron ionization (EI) mass spectra were acquired over the mass range 35-350 Da.

\subsection{Nuclear Magnetic Resonance}

All ${ }^{13} \mathrm{C}$-NMR spectra were recorded on a Bruker AVANCE 400 Fourier transform spectrometer (Bruker, Wissembourg, France) operating at $100.623 \mathrm{MHz}$ for ${ }^{13} \mathrm{C}$, equipped with a $5 \mathrm{~mm}$ probe, in $\mathrm{CDCl}_{3}$, with all shifts referred to via an internal TMS. The following parameters were used: pulse width $=4 \mu \mathrm{s}$ (flip angle $45^{\circ}$ ); relaxation delay D1 $=0.1 \mathrm{~s}$, acquisition time $=2.7 \mathrm{~s}$ for a $128 \mathrm{~K}$ data table with a spectral width of $25,000 \mathrm{~Hz}(250 \mathrm{ppm})$; CPD mode decoupling; digital resolution $=0.183 \mathrm{~Hz} / \mathrm{pt}$. The number of accumulated scans was 3000 for each sample or fraction $\left(40 \mathrm{mg}\right.$, when available, in $0.5 \mathrm{~mL}$ of $\mathrm{CDCl}_{3}$ ). 


\subsection{Identification of Individual Components}

Identification of the individual components was carried out: (i) by a comparison of their GC retention indices on apolar and polar columns, with those of reference compounds [25,37]; (ii) by computer matching against commercial mass spectral libraries [37-39]; (iii) by a comparison of the signals in the ${ }^{13} \mathrm{C}-\mathrm{NMR}$ spectra of the samples and fractions with those of reference spectra compiled in the laboratory spectral library, with the help of a laboratory-made software $[15,22]$. This method allowed for the identification of individual components of the essential oil at contents as low as $0.4-0.5 \%$.

\subsection{Statistical Analysis}

The chemical compositions of 47 leaf essential oil samples from I. dewevrei were submitted to a hierarchical cluster analysis (HCA) and principal component analysis (PCA) using XLSTAT software (Addinsoft, Paris, France) [40]. Only constituents in a concentration higher than $1.0 \%$ were used as variables for the PCA analysis. The aptitude of the complete correlation matrix was checked by the Kaiser-Meyer-Olkin criterion. The HCA and dendrogram were made with dissimilarity matrices calculated in Euclidean distance, and the average link was the aggregation method systematically chosen.

\subsection{In Vitro Anti-Inflammatory Capacity of Isolona dewevrei Leaf Essential Oil}

The in vitro anti-inflammatory capacity of $I$. dewevrei leaf essential oil was evaluated by an in vitro lipoxygenase inhibition assay [41-43]. The in vitro analysis for lipoxygenase inhibitory activity was performed using Lipoxidase type I-B (Lipoxygenase, LOX, EC 1.13.11.12) from Glycine max (soybean) purchased from Sigma-Aldrich Chimie (Saint-Quentin-Fallavier, France). It was determined by the kinetic mode of the spectrophotometric determination method, which was performed by recording the rate of change in absorbance at $234 \mathrm{~nm}$. Indeed, the increase in absorbance at $234 \mathrm{~nm}$ was due to the formation of 13-hydroperoxides of linoleic acid (substrate used for LOX inhibition activity assay) [41-43].

A stock solution of LOX was prepared by dissolving around 5.7 units/mL of LOX in PBS (Phosphate Buffer Solution; 1 unit corresponding to $1 \mu \mathrm{mol}$ of hydroperoxide formed per min). Four concentrations of I. dewerrei leaf essential oil sample (S44) in dimethylsulfoxide (DMSO) were tested as the inhibitor solution for the LOX inhibition activity assay: $0.005,0.010,0.015$ and $0.020 \mathrm{mg} / \mathrm{mL}$.

The LOX inhibition assays were performed by mixing $10 \mu \mathrm{L}$ of LOX solution with $10 \mu \mathrm{L}$ of inhibitor solution in $970 \mu \mathrm{L}$ of boric acid buffer $(50 \mathrm{mM}$; $\mathrm{pH} 9.0)$ and incubating them briefly at room temperature. The reaction started by the addition of $10 \mu \mathrm{L}$ of substrate solution (Linoleic acid, $25 \mathrm{mM}$ ), and the velocity was recorded for $30 \mathrm{~s}$ at $234 \mathrm{~nm}$. One assay was measured in the absence of the inhibitor solution, and one assay was measured with DMSO mixed with distilled water $(99.85 \%$ of DMSO in distilled water) which made it possible to eliminate the inhibition effect of DMSO. No inhibitor effect of DMSO on the LOX activity was detected, and the LOX activity measured without inhibitor solution was considered as a control (100\% enzymatic reaction). All assays were performed in triplicate. The percentage of lipoxygenase inhibition was calculated according to the equation:

$$
\text { Inhibition } \%=\left(\mathrm{V}_{\text {0control }}-\mathrm{V}_{\text {0sample }}\right) \times 100 / \mathrm{V}_{\text {0control }}
$$

$\mathrm{V}_{\text {0control }}$ is the activity of LOX in the absence of the inhibitor solution, and $\mathrm{V}_{\text {0sample }}$ is the activity of LOX in the presence of the inhibitor solution [43]. The $\mathrm{IC}_{50}$ was calculated by the concentration of I. dewerorei leaf essential oil in DMSO inhibiting $50 \%$ of LOX activity.

\section{Conclusions}

The chemical variability of the leaf essential oil from I. dewevrei growing wild in Côte d'Ivoire was investigated through 47 oil samples. A combination of chromatographic (CC, GC(RI)) and spectroscopic (GC/MS, $\left.{ }^{13} \mathrm{C}-\mathrm{NMR}\right)$ techniques was used to determine the samples' chemical compositions. One hundred and thirteen constituents accounting for 
90.8-98.9\% of the whole sample compositions were identified, and the main components varied drastically from sample to sample. Therefore, the 47 oil compositions were submitted to hierarchical cluster and principal components analyses, which evidenced three distinct chemical groups, each dividing into two subgroups. The Subgroup I-A was dominated by $(Z)$ - $\beta$-ocimene, $\beta$-eudesmol, germacrene $D$ and $(E)$ - $\beta$-ocimene, while $(10 \beta H)-1 \beta, 8 \beta$ oxido-cadina-4-ene, santalenone, trans- $\alpha$-bergamotene and trans- $\beta$-bergamotene were the main compounds of Subgroup I-B. The prevalent constituents of Subgroup II-A were germacrene $\mathrm{B},(E)$ - $\beta$-caryophyllene, $(5 \alpha \mathrm{H}, 10 \beta \mathrm{Me})$-6,12-oxido-elema-1,3,6,11(12)-tetraene and $\gamma$-elemene. The Subgroup II-B displayed germacrene B, germacrene D and (Z)- $\beta$ ocimene as the majority compounds. Germacrene D was the most abundant constituent of Group III, followed in Subgroup III-A by $(E)-\beta$-caryophyllene, $(10 \beta \mathrm{H})-1 \beta$, $8 \beta$-oxidocadina-4-ene, germacrene D-8-one, and then in Subgroup III-B by (Z)- $\beta$-ocimene and $(E)$ $\beta$-ocimene. Compounds bearing the eudesmane skeleton characterized Subgroup I-A and also Subgroup III-B to a lesser extent. Likewise, santalane, bergamotane and bisabolane skeletons were markers of Subgroup I-B, while the elemane skeleton was specific to Group II. Group III markers were oxygenated germacrane and cadinane compounds. Although genetic factors could not be completely excluded, the observed qualitative and quantitative chemical variability of the leaf essential oil from $I$. dewevrei could be related to mostly phenology and season, then harvest site to a lesser extent. A leaf oil sample (S44) was tested for its lipoxygenase inhibition ability. The oil $\mathrm{IC}_{50}$ value $(0.020 \pm 0.005 \mathrm{mg} / \mathrm{mL})$ was slightly higher than the non-competitive lipoxygenase inhibitor NDGA $\mathrm{IC}_{50}$ value $(0.013 \pm 0.003 \mathrm{mg} / \mathrm{mL})$. Therefore, this leaf essential oil exhibited significant in vitro antiinflammatory potential.

Supplementary Materials: The following are available online, Table S1 (Chemical composition of the 47 leaf essential oil samples from Isolona dewevrei) and Table S2 (Plant material, essential oil extraction and climate data).

Author Contributions: Conceptualization, D.A.K., J.B.B. and F.T.; methodology, D.A.K., J.B.B. and F.T.; software, D.A.K. and M.P.; validation, J.B.B. and F.T.; formal analysis, D.A.K.; data analysis, D.A.K., A.C.L.K. and D.B.; essential oil investigation, D.A.K., A.C.L.K. and D.B.; in vitro antiinflammatory activity investigation, V.B.; writing—original draft preparation, D.A.K., J.B.B. and F.T.; writing-review and editing, D.A.K., J.B.B. and F.T.; visualization, D.A.K. and M.P.; supervision, J.B.B. and F.T. All authors have read and agreed to the published version of the manuscript.

Funding: This research received no external funding.

Institutional Review Board Statement: Not applicable.

Informed Consent Statement: Not applicable.

Data Availability Statement: The data presented in this study are available in Supplementary Materials.

Acknowledgments: The authors gratefully acknowledge the University of Corsica for providing a post-doctoral grant to D. A. Kambiré. We acknowledge J. Assi and H. Téré for their valuable help in the plant identification, and Marc Gibernau for the statistical data analysis.

Conflicts of Interest: The authors declare no conflict of interest.

Sample Availability: Samples of the essential oils are available from the authors.

\section{References}

1. Couvreur, T.L.P. Revealing the Secrets of African Annonaceae: Systematics, Evolution and Biogeography of the Syncarpous Genera Isolona and Monodora. Ph.D. Thesis, Wageningen University, Wageningen, The Netherlands, $2008 ;$ p. 294.

2. Aké, A.L. Flore de Côte D'ivoire: Étude Descriptive et Biogéographique avec Quelques Notes Ethno-Botaniques. Ph.D. Thesis, Université d'Abidjan, Abidjan, Côte d'Ivoire, 1984; p. 1206.

3. Achenbach, H.; Löwell, M. Constituents of Isolona maitlandii. Phytochemistry 1995, 40, 967-973. [CrossRef]

4. Makangara, J.J.; Henry, L.; Jonker, S.A.; Nkunya, M.H.H. The caulindoles: Dimeric prenylindoles from Isolona cauliflora. Phytochemistry 2004, 65, 227-232. [CrossRef] [PubMed]

5. Nkunya, M.H.H.; Makangara, J.J.; Jonker, S.A. Prenylindoles from Tanzanian Monodora and Isolona species. Nat. Prod. Res. 2004, 18, 253-258. [CrossRef] 
6. Hocquemiller, R.; Cabalion, P.; Bouquet, A.; Cavé, A.; Janot, M.-M. L'isopiline, nouvel alcaloïde aporphinique isolé de 1'Isolona pilosa Diels, Annonacées. C. R. Acad. Sci. Paris Sér. C 1977, 285, 447-450.

7. Hocquemiller, R.; Cabalion, P.; Bruneton, J.; Cavé, A. Alcaloïdes des Annonacées XXIII. Alcaloïdes des écorces d'Isolona campanulata Engler et Diels. Plantes Méd. Phytothér. 1978, 12, 230-234.

8. Hocquemiller, R.; Cabalion, P.; Fournet, A.; Cavé, A. Alcaloïdes des Annonacées XLIX. Alcaloïdes d'Isolona hexaloba, I. zenkeri et I. pilosa. Planta Med. 1984, 50, 23-25. [CrossRef] [PubMed]

9. Leboeuf, M.; Cavé, A.; Bhaumik, P.K.; Mukherjee, B.; Mukherjee, R. The phytochemistry of the Annonaceae. Phytochemistry 1982, 21, 2783-2813. [CrossRef]

10. Okpekon, A.T. Étude Chimique et Biologique de Plantes Ivoiriennes Utilisées en Médecine Traditionnelle en tant Qu'antiparasitaires: Afromomum sceptrum K. Schum. (Zingiberaceae), Bridelia ferruginea Benth. (Euphorbiaceae), Isolona cooperi Hutch. \& Dalz. et Uvaria afzelii Sc. Elliot (Annonaceae). Ph.D. Thesis, Université Paris-Sud, France-Université de Cocody-Abidjan, Côte d'Ivoire, Paris, France, 2006; p. 283.

11. Kabran, A.F. Étude Phytochimique de Plantes Ivoiriennes à Activité Antiparasitaire. Ph.D. Thesis, Université Paris-Sud, FranceUniversité de Cocody-Abidjan, Côte d'Ivoire, Paris, France, 2013; p. 290.

12. Boti, J.B.; Koukoua, G.; N'Guessan, Y.T.; Muselli, A.; Bernardini, A.F.; Casanova, J. Composition of the leaf, stem bark and root bark oils of Isolona cooperi investigated by GC (retention index), GC-MS and ${ }^{13}$ C-NMR spectroscopy. Phytochem. Anal. 2005, 16, 357-363. [CrossRef] [PubMed]

13. Boti, J.B.; Yao, A.P.; Koukoua, G.; N'Guessan, Y.T.; Casanova, J. Components and chemical variability of Isolona campanulata Engler \& Diels leaf oil. Flavour Fragr. J. 2006, 21, 166-170. [CrossRef]

14. Kambiré, D.A.; Boti, J.B.; Ouattara, Z.A.; Yapi, A.T.; Nelly, B.; Bighelli, A.; Tomi, F. Chemical composition of root and stem bark essential oils from Ivorian Isolona dewevrei: Structural elucidation of a new natural germacrone. Nat. Prod. Res. 2021, 1-7. [CrossRef] [PubMed]

15. Kambiré, D.A.; Boti, J.B.; Ouattara, Z.A.; Yapi, A.T.; Bighelli, A.; Tomi, F.; Casanova, J. Leaf essential oil from Ivoirian Isolona dewevrei (Annonaceae): Chemical composition and structure elucidation of four new natural sesquiterpenes. Flavour Fragr. J. 2021, 36, 22-33. [CrossRef]

16. Kambiré, D.A.; Boti, J.B.; Yapi, T.A.; Ouattara, Z.A.; Bighelli, A.; Casanova, J.; Tomi, F. New Natural Oxygenated Sesquiterpenes and Chemical Composition of Leaf Essential Oil from Ivoirian Isolona dewevrei (De Wild. \& T. Durand) Engl. \& Diels. Molecules 2020, 25, 5613. [CrossRef]

17. Nea, F.; Kambiré, D.A.; Genva, M.; Tanoh, E.A.; Wognin, E.L.; Martin, H.; Brostaux, Y.; Tomi, F.; Lognay, G.C.; Tonzibo, Z.F.; et al. Composition, Seasonal Variation, and Biological Activities of Lantana camara Essential Oils from Côte d'Ivoire. Molecules 2020, 25, 2400. [CrossRef] [PubMed]

18. Kambiré, D.A.; Boti, J.B.; Yapi, A.T.; Ouattara, Z.A.; Paoli, M.; Bighelli, A.; Tomi, F.; Casanova, J. Composition and intraspecific chemical variability of leaf essential oil of Laggera pterodonta (DC.) Sch. Bip. ex Oliv. from Côte d'Ivoire. Chem. Biodivers. 2020, 17, e1900504. [CrossRef]

19. Kambiré, D.A.; Yapi, A.T.; Boti, J.B.; Ouattara, Z.A.; Tonzibo, Z.F.; Filippi, J.J.; Bighelli, A.; Tomi, F. Two new eudesman-4 $\alpha$-ol epoxides from the stem essential oil of Laggera pterodonta from Côte d'Ivoire. Nat. Prod. Res. 2020, 34, 2765-2771. [CrossRef] [PubMed]

20. Kambiré, D.A.; Boti, J.B.; Filippi, J.J.; Tonzibo, Z.F.; Tomi, F. Characterization of a new epoxy-hydroxycarvotanacetone derivative from the leaf essential oil of Laggera pterodonta from Côte d'Ivoire. Nat. Prod. Res. 2019, 33, 2109-2112. [CrossRef] [PubMed]

21. Kambiré, D.A.; Yapi, A.T.; Boti, J.B.; Garcia, G.; Tomi, P.; Bighelli, A.; Tomi, F. Chemical composition of leaf essential oil of Piper umbellatum and aerial part essential oil of Piper guineense from Côte d'Ivoire. Nat. Prod. Commun. 2019, 14, 1-8. [CrossRef]

22. Tomi, F.; Bradesi, P.; Bighelli, A.; Casanova, J. Computer-aided identification of individual components of essential oils using carbon-13 NMR spectroscopy. J. Magn. Reson. Anal. 1995, 1, 25-34.

23. Baldovini, N.; Tomi, F.; Casanova, J. Identification and quantitative determination of furanodiene, a heat-sensitive compound, in essential oil by ${ }^{13}$ C-NMR. Phytochem. Anal. 2001, 12, 58-63. [CrossRef]

24. Ouattara, Z.A.; Boti, J.B.; Ahibo, A.C.; Casanova, J.; Tomi, F.; Bighelli, A. Analysis of Cleistopholis patens leaf and trunk bark oils using combined GC-Flame Ionisation Detection, GC-Retention Index, GC-MS and ${ }^{13}$ C-NMR. Phytochem. Anal. 2013, 24, 574-580. [CrossRef] [PubMed]

25. Terpenoids Library Website. Available online: https://massfinder.com/wiki/Terpenoids_Library_List (accessed on 6 October 2021).

26. Baharum, S.N.; Bunawan, H.; Ghani, M.A.; Mustafa, W.A.W.; Noor, N.M. Analysis of the chemical composition of the essential oil of Polygonum minus Huds. using two-dimensional gas chromatography-Time of flight mass spectrometry (GC-TOF-MS). Molecules 2010, 15, 7006-7015. [CrossRef]

27. Cavalli, J.-F.; Tomi, F.; Bernadini, A.-F.; Casanova, J. Composition and chemical variability of the bark oil of Cedrelopsis grevei H. Baillon from Madagascar. Flavour Fragr. J. 2003, 18, 532-538. [CrossRef]

28. Edris, A.E.; Chizzola, R.; Franz, C. Isolation and characterization of the volatile aroma compounds from the concrete headspace and the absolute of Jasminum sambac (L.) Ait. (Oleaceae) flowers grown in Egypt. Eur. Food Res. Technol. 2008, 226, 621-626. [CrossRef]

29. Weyerstahl, P.; Marschall, H.; Wahlburg, H.-C.; Christiansen, C.; Rustaiyan, A.; Mirdjalili, F. Constituents of the essential oil of Pulicaria gnaphalodes (Vent.) Boiss. from Iran. Flavour Fragr. J. 1999, 14, 121-130. [CrossRef] 
30. Paolini, J.; Tomi, P.; Bernardini, A.-F.; Bradesi, P.; Casanova, J.; Kaloustian, J. Detailed analysis of the essential oil from Cistus albidus L. by combination of GC/RI, GC/MS and ${ }^{13}$ C-NMR spectroscopy. N. Z. J. Agric. Res. 2008, 22, 1270-1278. [CrossRef] [PubMed]

31. Carrasco, A.; Ortiz-Ruiz, V.; Martinez-Gutierrez, R.; Tomas, V.; Tudela, J. Lavandula stoechas essential oil from Spain: Aromatic profile determined by gas chromatography-mass spectrometry, antioxidant and lipoxygenase inhibitory bioactivities. Ind. Crops Prod. 2015, 73, 16-27. [CrossRef]

32. Cutillas, A.-B.; Carrasco, A.; Martinez-Gutierrez, R.; Tomas, V.; Tudela, J. Thyme essential oils from Spain: Aromatic profile ascertained by GC-MS and their antioxidant, anti-lipoxygenase and antimicrobial activities. J. Food. Drug Anal. 2018, 26, 529-544. [CrossRef]

33. Whitman, S.; Gezginci, M.; Timmermann, B.N.; Holman, T.R. Structure-activity relationship studies of nordihydroguaiaretic acid inhibitors toward soybean, 12-human, and 15-human lipoxygenase. J. Med. Chem. 2002, 45, 2659-2661. [CrossRef] [PubMed]

34. Jazet Dongmo, P.M.; Kuate, J.; Ngouana, V.; Damesse, F.; Tchinda Sonwa, E.; Amvam Zollo, P.H.; Menut, C. Comparaison des propriétés anti-radicalaires et anti-inflammatoires des huiles essentielles de Citrus reticulata var. madagascar et Citrus sinensis var. casagrande du Cameroun. Fruits 2008, 63, 201-208.

35. Kamatou, G.P.P.; Viljoen, A.M.; Gono-Bwalya, A.B.; van Zyl, R.L.; van Vuuren, S.F.; Lourens, A.C.U.; Başer, K.H.C.; Demirci, B.; Lindsey, K.L.; van Staden, J.; et al. The in vitro pharmacological activities and a chemical investigation of three South African Salvia species. J. Ethnopharmacol. 2005, 102, 382-390. [CrossRef]

36. Cachet, T.; Brevard, H.; Chaintreau, A.; Demyttenaere, J.; French, L.; Gassenmeier, K.; Joulain, D.; Koenig, T.; Leijs, H.; Liddle, P.; et al. IOFI recommended practice for the use of predicted relative-response factors for the rapid quantification of volatile flavouring compounds by GC-FID. Flavour Fragr. J. 2016, 31, 191-194. [CrossRef]

37. König, W.A.; Hochmuth, D.H.; Joulain, D. Terpenoids and Related Constituents of Essential Oils. Library of MassFinder 2.1; Institute of Organic Chemistry: Hamburg, Germany, 2001.

38. National Institute of Standards and Technology. PC Version of the Mass Spectral Library; Norwalk: Connecticut, CT, USA, 2014.

39. Adams, R.P. Identification of Essential Oils Components by Gas Chromatography/Mass Spectroscopy, 4th ed.; Allured: Carol Stream, IL, USA, 2001; p. 455.

40. Legendre, P.; Legendre, L. Numerical Ecology, 2nd ed.; Elsevier Science: Amsterdam, The Netherlands, $1998 ;$ p. 853.

41. Eshwarappa, R.S.B.; Ramachandra, Y.L.; Subaramaihha, S.R.; Subbaiah, S.G.P.; Austin, R.S.; Dhananjaya, B.L. Anti-Lipoxygenase activity of leaf gall extracts of Terminalia chebula (Gaertn.) Retz. (Combretaceae). Pharmacogn. Res. 2016, 8, 78-82. [CrossRef]

42. Srivastava, P.; Vyas, V.K.; Variya, B.; Patel, P.; Qureshi, G.; Ghate, M. Synthesis, anti-inflammatory, analgesic, 5-lipoxygenase (5-LOX) inhibition activities, and molecular docking study of 7-substituted coumarin derivatives. Bioorg. Chem. 2016, 67, 130-138. [CrossRef] [PubMed]

43. Bayala, B.; Bassole, I.H.; Gnoula, C.; Nebie, R.; Yonli, A.; Morel, L.; Figueredo, G.; Nikiema, J.-B.; Lobaccaro, J.-M.; Simpore, J. Chemical composition, antioxidant, anti-Inflammatory and anti-proliferative activities of essential oils of plants from Burkina Faso. PLoS ONE 2014, 9, e92122. [CrossRef] [PubMed] 